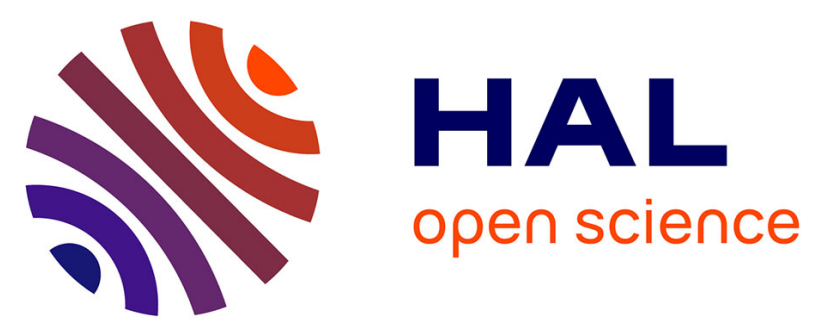

\title{
Large-Signal Stabilization of Power Converters Cascaded Input Filter Using Adaptive Energy Shaping Control
}

\author{
Shengzhao Pang, Saeid Aghaei Hashjin, Babak Nahid-Mobarakeh, Serge
}

Pierfederici, Yigeng Huangfu, Guangzhao Luo, Fei Gao

\section{- To cite this version:}

Shengzhao Pang, Saeid Aghaei Hashjin, Babak Nahid-Mobarakeh, Serge Pierfederici, Yigeng Huangfu, et al.. Large-Signal Stabilization of Power Converters Cascaded Input Filter Using Adaptive Energy Shaping Control. IEEE Transactions on Transportation Electrification, 2020, 7 (2), pp.838-853. 10.1109/TTE.2020.3021954 . hal-03192933

\section{HAL Id: hal-03192933 \\ https://hal.univ-lorraine.fr/hal-03192933}

Submitted on 10 May 2021

HAL is a multi-disciplinary open access archive for the deposit and dissemination of scientific research documents, whether they are published or not. The documents may come from teaching and research institutions in France or abroad, or from public or private research centers.
L'archive ouverte pluridisciplinaire HAL, est destinée au dépôt et à la diffusion de documents scientifiques de niveau recherche, publiés ou non, émanant des établissements d'enseignement et de recherche français ou étrangers, des laboratoires publics ou privés.

\section{(c)(1)}

Distributed under a Creative Commons Attribution| 4.0 International License 


\title{
Large-Signal Stabilization of Power Converters Cascaded Input Filter Using Adaptive Energy Shaping Control
}

\author{
Shengzhao Pang, Student Member, IEEE, Saeid Aghaei Hashjin, Babak Nahid-Mobarakeh, Senior \\ Member, IEEE, Serge Pierfederici, Yigeng Huangfu, Senior Member, IEEE, Guangzhao Luo, Member, \\ IEEE, and Fei Gao, Senior Member, IEEE
}

\begin{abstract}
Series connection of input filters with static converters might lead to instability. However, the input filter has rarely taken into account in the stable design of power converters. Indeed, an input $L C$ filter cascaded with a converter can become unstable, and so even if the converter is regulated by a tight controller ensuring its stability alone. This fact is due to the interactions between the filter and the converter. To tackle the instability potential, an Adaptive Energy Shaping Control (AESC), which is based on the Interconnection and Damping Assignment Passivity-Based Control (IDA-PBC), is addressed in this paper to regulate the cascaded system and achieve the following attractive features: 1) The input filter's dynamics are considered in the control law, so the interactions between the filter and the converter are taken into account during the controller design; 2) The influence between several subsystems, put in cascade, is considered by the proposed method and the new large-signal stability proof is given accordingly. Simulation and experimental results from a $3.5 \mathrm{~kW} 270 \mathrm{~V}-200 \mathrm{~V}$ buck converter cascaded with an input filter under different load conditions, i.e. Constant Impedance Load (CIL), Constant Current Load (CCL), and Constant Power Load (CPL), are presented to demonstrate the proposed approach.

Index Terms-Lyapunov-based stability, input filter, dc-dc power converter, large-signal stability, dc microgrids, electrified transportation, comparison, adaptive energy shaping control.
\end{abstract}

\section{INTRODUCTION}

DC distribution power system in the form of dc microgrid has received increasing attention, especially in electrified transportation applications such as More Electric Aircraft (MEA), Electric Vehicles (EVs), ships, and submarines [1], [2], [3].

S. Pang is with the Groupe de Recherche en Energie Electrique de Nancy, École Nationale Supérieure d'Électricité et de Mécanique, Université de Lorraine, Nancy 54505, France (e-mail: shengzhao.pang@univ-lorraine.fr).

$\mathrm{S}$. Aghaei Hashjin is with the Groupe de Recherche en Energie Electrique de Nancy, École Nationale Supérieure d'Électricité et de Mécanique, Université de Lorraine, Nancy, France, and also with Ecole Spéciale de Mécanique et d'Electricité, Paris, France (e-mail: aghaei-hashjin.saeid@univ-lorraine.fr).

B. Nahid-Mobarakeh is with the Department of Electrical and Computer Engineering, McMaster University, Hamilton, Ontario L8S 4L8, Canada, and also with the Groupe de Recherche en Energie Electrique de Nancy, École
In this application, instability is still a key issue in the design of $\mathrm{dc}$ microgrids. These systems, consisting of multiple cascaded or parallel converters, can suffer from instability, even when individual converters are stable alone [4], [5]. This instability can be induced by the interactions between the individually designed converters or the influences from the poorly damped input $L C$ filter and tightly regulated loads, e.g., constant power loads [6], [7].

A typical architecture for the on-board dc microgrid is shown in Fig. 1 [3], [8]. The source-connected converter can be the dcdc converter, which delivers energy from the dc source or energy storage element, e.g., battery, ultra-capacitor, etc. It can also be an ac-dc converter for transferring energy from the ac microgrid or the generator [9]. Since the dc-link voltage is not fit in well with loads, the load-connected converters are used to regulate the voltage level [10]. The output $L C$ filter of the source-connected converter and the input $L C$ filter of the loadconnected converter are implemented to protect the dc-bus. More specifically, the output filter is mainly used to limit harmonic voltage content on the dc-bus. The input filter is mainly used to respect the current ripple constraint on the dcbus [11]. Such filters are usually poorly damped for size/weight optimization or loss reduction consideration [12].

The types of loads acting on the dc microgrid can be broadly classified as Constant Impedance Load (CIL), Constant Current Load (CCL), and Constant Power Load (CPL) [13]. Usually, the negative incremental impedance property of CPL introduces a destabilizing effect on the operation with its upstream circuit, e.g., typically $L C$ filters and dc-dc converters [14].

Nationale Supérieure d'Électricité et de Mécanique, Université de Lorraine, Nancy 54505, France (e-mail: babak.nahid@univ-lorraine.fr).

$\mathrm{S}$. Pierfederici is with the Laboratoire d'Energétique et de Mécanique Théorique et Appliquée, École Nationale Supérieure d'Électricité et de Mécanique, CNRS-Université de Lorraine, Nancy 54504, France (e-mail: serge.pierfederici@univ-lorraine.fr).

Y. Huangfu and G. Luo are with the School of Automation, Northwestern Polytechnical University, Xi'an 710072, China (e-mail: yigeng@nwpu.edu.cn; guangzhao.luo@nwpu.edu.cn).

F. Gao is with the FEMTO-ST Institute and the FCLAB, Universite Bourgogne Franche-Comté, UTBM, Belfort, France (e-mail: fei.gao@utbm.fr). 


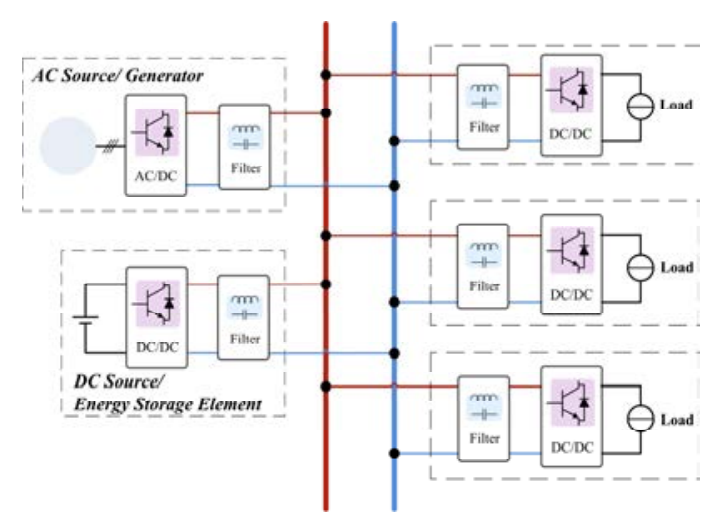

Fig. 1 The typical architecture for the on-board dc microgrid.

To help the stabilization, various methods have been introduced and can be classified into two groups, i.e., passive approaches [15] and active approaches [7]. Passive dampers use passive circuit components (resistors, inductors, and/or capacitors) and are employed to stabilize the system by modifying the input/output impedance of the system [16]. However, losses, volume, and weight are increased due to these types of dampers.

To overcome the abovementioned drawback, different active approaches have been proposed in the literature. Some active impedance-based methods are also called virtual impedance techniques, which adjust the output impedance of the source converter or the input impedance of the load converter to meet the stability requirements [17], [18], [19], [20]. The method is developed based on transfer functions and impedance specifications. For example, [17]-[19] introduce the virtual impedance at the source converter. [20] proposes a virtual impedance at the load converter to stabilize the cascaded system. The advantage of this kind of method is that it gives the specific physical meaning to the active approach. It helps the engineer to comprehend and utilize this method. However, if the source converter is an $L C$ filter, the virtual impedance cannot be applied to the source converter, because the $L C$ filter is uncontrollable and the impedance is unchangeable [20]. Also, the implementation of the virtual impedance technique at the load converter may lead to the compromise of load performance [17]. Furthermore, in this group of methods, the original structure of the control system will be modified.

Moreover, some active stabilizers have been proposed which include injecting the stabilizing signal to the feedback control loop of the source converter [21] and/or the load converter [7]. These methods are usually developed based on the state-space model and studying the eigenvalues of the Jacobian matrix. There are three main types of the active stabilizers, including injecting the stabilizing signal into the outer-loop [22], the inner-loop [23], or directly to the duty cycle [7], [21]. When applying the stabilizing signal to the outer- or inner-loop, their performances are limited according to the bandwidth of the controller, especially the outer-loop bandwidth is relatively low in practice. Furthermore, the original structure of the control system is also modified. Stabilization occurs directly through the duty cycle and is the quickest stabilizing action that we can realize, but it might be sensitive to noise.
Besides, some auxiliary power electronic circuits between the source and load converter are introduced. For example, [24] proposes an adaptive active capacitor converter which is connected in parallel with the intermediate bus of the cascaded system. However, the number of auxiliary circuits increases with the number of cascaded systems and is therefore not suitable for the microgrid. Furthermore, as discussed in [15], the active approach would sometimes contradict with other control objectives.

Overall, all of the above approaches use the small-signal stability criteria. This latter is based on a linearized model around the operating point. However, when large-signal disturbances occur, the intrinsic nonlinearity of the power electronics system becomes inevitable so that the linearization is no longer valid. Then, when the system experiences large disturbances, the probability of losing instability will increase. Therefore, microgrids require a new stabilization method based on large-signal stability and can also guarantee the stability of the whole system.

Being aware of the abovementioned requirements, the Passivity-Based Control (PBC) offers an effective theoretical tool to achieve them. It can ensure the large-signal stability of the entire system by using the passivity and Lyapunov concepts. The large-signal stability of the whole system can be guaranteed by using the passivity property when several stable and passive ensured subsystems are integrated into the microgrid [25]. PBC has been widely used in many physical systems including power electronics converters, such as dc-dc converter [26], [27], [28], ac-ac converter [29], three-phase inverter [30], and solidstate transformer [31]. These controllers are based on EulerLagrange (EL) models or port-controlled Hamiltonian (PCH) models, which provide the generalized integrated structures for PBC. The Interconnection and Damping Assignment PassivityBased Control (IDA-PBC) is introduced by [32] as a branch of PBC, which is based on PCH models. However, these works do not take into account the input filter, which seriously affects the system dynamics. Also, they do not consider more complex configurations with several cascaded subsystems. To overcome these drawbacks, this paper proposes an Adaptive Energy Shaping Control (AESC) which is based on the IDA-PBC methodology.

The remaining of the paper is organized as follows. Section II shows stability problems in conventional IDA-PBC. Section III and Section IV present the proposed AESC with a design example. Some simulations carried out by MATLAB in Section $\mathrm{V}$ and experimental results are included in Section VI. It is worthy to note that some preliminary simulation results with a focus on the special case of CPL can be found in [14]. This paper is wrapped up with some concluding remarks in Section VII.

\section{PROBLEM FormULATION}

\section{A. Review of the Conventional IDA-PBC}

The IDA-PBC methodology based on PCH is first introduced in [32], and is detailed for dc-dc power converters in [25] and [28]. The final control law $u$ of the conventional IDA-PBC is calculated by the following expression, 


$$
\begin{gathered}
{[J(x)-R(x)] \frac{\partial H}{\partial x}(x)+\beta(x) u+\zeta} \\
=\left[J_{d}(x)-R_{d}(x)\right] \frac{\partial H_{d}}{\partial x}(x)
\end{gathered}
$$

The left-hand side of (1) is the open-loop system dynamics. The interconnection matrix $J(x)$ (skew-symmetric), dissipation matrix $R(x)$, coefficient matrix $\beta(x)$ and disturbance matrix $\zeta$ are all known matrices. $H(x)=\frac{1}{2} x^{T} Q x \geq 0$ denotes the total stored energy called Hamiltonian function. Matrix $Q$ stands for the energy storage elements in the system. The right-hand side of (1) is the closed-loop system dynamics. The desired interconnection matrix $J_{d}(x)$ and damping matrix $R_{d}(x)$ are selected by the designer. $H_{d}(x)=\frac{1}{2}\left(x-x_{d}\right)^{T} Q\left(x-x_{d}\right) \geq 0$ is the closed-loop Hamiltonian function and $x_{d}$ is the desired operating point [25]. The damping matrix $R_{d}(x)$ can be designed based on the natural dissipation matrix, i.e. $R_{d}(x)=$ $R(x)+R_{a}(x)$. If no extra damping assigned, i.e. $R_{a}(x)=0$, the control law is derived from natural damping [32].

The stability of the system is guaranteed by selecting the closed-loop Hamiltonian function as Lyapunov candidate function, i.e. $V=H_{d}(x)$. It is worthwhile to note that, if the desired operating point $x_{d}$ is constant, the total time derivative of this candidate function can be written as,

$$
\dot{V}=\left[\frac{\partial H_{d}(x)}{\partial x}\right]^{T} \dot{x}=-\left[\frac{\partial H_{d}(x)}{\partial x}\right]^{T} R_{d} \frac{\partial H_{d}(x)}{\partial x} \leq 0
$$

As a result, $H_{d}(x)$ does not increase and can be qualified as a Lyapunov function. The stability of the operating point is guaranteed.

Moreover, as can be seen from (1), the control law $u$ is the only unknown in the equation. In power electronic devices, the current and voltage in the energy storage element are usually considered to be state variables. Thus, there are at least two state variables in a common dc-dc converter. As a consequence, equation (1) may have the possibility of no unique solution. In [28], the authors use only the partial equation of the $\mathrm{PCH}$ models to derive the final control law. In fact, the Hamiltonian function of the $\mathrm{PCH}$ model includes all the internal energy accumulations in the system. Afterward, the stability proof considers all the equations in the closed-loop PCH framework. So, the final control law should include all the state variables and serve as the unique solution to all the equations. To this end, the authors in [25] introduce an adaptive closed-loop interconnection matrix to give the additional degree of freedom for building up the internal links in the $\mathrm{PCH}$ system, and to guarantee that equation (1) has a unique solution.

\section{B. Stability Problem due to the Cascade Structure}

However, conventional IDA-PBC does not work when several cascaded subsystems are employed in the microgrid. In the conventional IDA-PBC, the desired operating point $x_{d}$ is related to the input disturbance $\zeta$, i.e. input voltage $V_{s}$ and load current $i_{\text {Load }}$ [25]. In a single dc-dc converter, the input voltage is constant and the load current is supposed to not vary so much. Therefore, $x_{d}$ is considered as a constant or is supposed to change very slowly. This is the condition on which the stability proof given in (2) depends. Unfortunately, when several dc-dc converters (can be considered as several subsystems) are cascaded into the microgrid, this assumption is no longer satisfied. Considering the topology structure shown in Fig. 2, the input voltage of subsystem 2 is actually the output voltage of subsystem 1 . Similarly, the load current of subsystem 2 is exactly the input current of subsystem 3 .

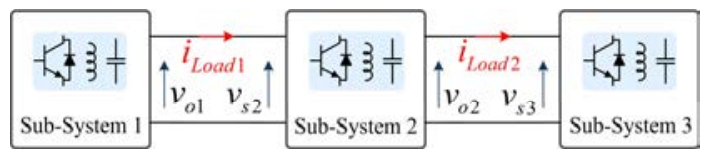

Fig. 2 Typical structure of cascaded systems.

Thus, $x_{d}$ depends on the neighbor subsystem's state variables in the cascaded system. It varies according to actual operating conditions, and its variations can no longer be ignored. The derivative of Lyapunov candidate function with respect to time results in

$$
\dot{V}=\left[\frac{\partial H_{d}(x)}{\partial x}\right]^{T} \dot{x}+\left[\frac{\partial H_{d}(x)}{\partial x_{d}}\right]^{T} \dot{x}_{d}
$$

It can clearly be seen that the proof of stability given in (2) will no longer apply, and the cascaded system in dc microgrids requires a new perspective for control and analysis.

\section{Stability Problem due to the Input Filter}

In the literature on the conventional IDA-PBC applied to dcdc converters, the authors do not usually consider the effect of the input $L C$ filter which closely disturbs the system dynamics. Indeed, it is known that the interaction between a poorly damped $L C$ filter (without extra damping) and a dc-dc converter may degrade the stability margins and even make the system unstable under some conditions [7]. Furthermore, as can be seen from the conclusion of [20], the uncontrollable $L C$ filter in the cascaded system is the most severe case in terms of stability.

Here, we reappear the conventional IDA-PBC given in [25] with the additional poorly damped input $L C$ filter. The load power steps from $1 \mathrm{~kW}$ to $2.5 \mathrm{~kW}$. The simulation results are shown in Fig. 3, where the system loses stability after the load power step. In the dc microgrid application, the $L C$ filter is directly connected to the dc-bus. This typical instability caused by the interaction between the $L C$ filter and the converter can affect the stability of the dc-bus, which in turn affects other loads in the de microgrid.

It is worthwhile to note that this instability problem can be avoided by damping the filter (adding resistors, inductors, and/or capacitors) according to the impedance specification [16]. However, losses, volume, and weight increase as a result. Therefore, this approach is not suitable for electrified transportation systems.

In the next section, An AESC law is proposed to address the above stability issues mentioned in paragraphs II.B and II.C. The variables of the input filter - power converter and their interactions will be taken into account into the controller. 

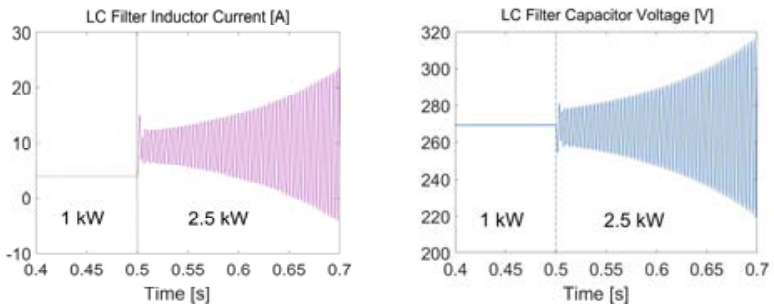

Fig. 3 Simulations results of the dc-dc buck converter cascaded with the input $L C$ filter when the conventional IDA-PBC law in [25] is used.

\section{Proposed Adaptive EnERGy Shaping Control: A GENERALIZED DESIGN}

As mentioned earlier, the desired operating point $x_{d}$ cannot be considered constant in the cascaded system. Let's introduce the tracking error $e$ and its variation $\dot{e}$ as follows

$$
\begin{aligned}
& e=x-x_{d} \\
& \dot{e}=\dot{x}-\dot{x}_{d}
\end{aligned}
$$

The closed-loop Hamiltonian function can be further expressed as

$$
H_{d}(e)=\frac{1}{2} e^{T} Q e
$$

Now, we define the desired closed-loop tracking error dynamics as

$$
\dot{e}=\left[J_{d}-R_{d}\right] \frac{\partial H_{d}}{\partial e}(e)
$$

The closed-loop Hamiltonian function $H_{d}(e)$ is considered as the Lyapunov candidate function. When $x \neq x_{d}, V=H_{d}>$ 0 . The total time derivative of $V$ is obtained in the following form

$$
\begin{gathered}
\dot{V}=\frac{1}{2}\left(\dot{e}^{T}\left[\frac{\partial H_{d}(e)}{\partial e}\right]+\left[\frac{\partial H_{d}(e)}{\partial e}\right]^{T} \dot{e}\right) \\
=-e^{T} Q^{T} R_{d} Q e<0
\end{gathered}
$$

As can be seen from (8), since the Lyapunov function $V$ is globally positive, radially unbounded, $R_{d}>0$, and $Q=Q^{T}>$ 0 , the time derivative of $V$ is globally negative in the domain of definition of the model. For the stable boundary, (8) is valid in the whole domain of definition of the model, i.e. the stability is global and not limited to small-signal variations around the operating point. Therefore, the desired operating point is global stable in the domain of definition of the model, or in the other words, the domain of attraction of the desired operating point is global, i.e. equal to the domain of validity of the model, so large-signal variations do not affect its stability. This statement is strictly held since its design is based on a proper Lyapunov function, so the large-signal stability of the whole system can be proved.

As for the selection of the tuning parameter, the following guidelines are used to choose the satisfactory parameter. The damping matrix $R_{d}$ is used to tune the dynamic performance and directly affects the set-point tracking. First, the controller is designed based on the Lyapunov stability theorem. According to (8), matrix $R_{d}$ should be positive-definite. Moreover, the proposed adaptive energy shaping control is a model-based control, that is, the closed-loop structure and control parameter should be designed according to the open-loop model and circuit parameters. If no extra damping is assigned $\left(R_{d}=R\right)$, the natural damping will be used for the system. It usually cannot meet dynamic requirements (overshoot and settling time). Then, we can increase the damping to meet the requirements (damping injection). This comes to place the eigenvalues of the matrix $-Q^{T} R_{d} Q$ in a domain in the left half complex plane where their real parts are less than $-\sigma_{0}$, where $\sigma_{0}>0$ is a stability margin defined by the designer. Here, the same guidelines as those employed for pole placement can be applied. Indeed, larger $\sigma_{0}$ means more control effort, so $\sigma_{0}$ should be selected within $\left[\sigma_{\min }, \sigma_{\max }\right]$ where $\sigma_{\min }$ is the least stability margin required and $\sigma_{\max }$ is to avoid duty cycles out of the range of $[0,1]$. Otherwise, the controller output $d$ saturates at 0 or 1 , and the passivity and stability proofs are no longer satisfied [25]. Another criterion is related to the digital implementation of the proposed control law limiting $\sigma_{\max }$. This latter cannot be selected very large unless the digital controller becomes unstable. These guidelines give only a range of values the designer can select for matrix $R_{d}$. Then, a trial and error or an optimization technique can be applied to set them. However, the optimization problem has to be clearly defined by setting first an objective function (stability margins, time response performances, control effort, robustness, etc.). This is worth another work to go in-depth with different design requirements. it is out of the scope of this paper where the main objective is to pave the way to stability analysis and stabilization of dc microgrids containing several power converters cascaded with their filters.

According to (5) and (7), the closed-loop system dynamics in $\mathrm{PCH}$ form can be described by

$$
\dot{x}=\left[J_{d}-R_{d}\right] \frac{\partial H_{d}}{\partial e}(e)+\dot{x}_{d}
$$

It is worthwhile to mention that the desired operating point $x_{d}$ is the reference of the state variables $x$. Therefore, it is only related to the disturbance $\zeta$ and independent from $x$. It yields

$$
\frac{\partial H_{d}(x)}{\partial x}=\frac{\partial H_{d}(e)}{\partial e}
$$

Based on (1), (9) and (10), the new control law $u$ can be calculated by,

$$
\begin{aligned}
{[J-R] \frac{\partial H}{\partial x}(x)+} & g(x) u+\zeta \\
& =\left[J_{d}-R_{d}\right] \frac{\partial H_{d}}{\partial x}(x)+\dot{x}_{d}
\end{aligned}
$$

The solution of (11) defines the control action $u$. To make it clear, a design example is given in the following section. 


\section{Design Example for the Buck Converter CAScaded WITH INPUT FILTER}

As mentioned before, thanks to the passivity property, the stability of the on-board microgrid can be guaranteed by studying the subsystem level stability. To do this latter, the PBC can be implemented and developed at the subsystem level. In this study, the proposed AESC strategy is applied to a subsystem which contains an input filter, a dc-dc converter, and a load. Such type of subsystems often appears in on-board dc microgrids.

The topological structure is given in Fig. 4. The $L C$ filter is connected between the dc-bus and the converter for filtering purposes. The $L C$ filter consists of an inductor $L_{f}$, a capacitor $C_{f}$, and equivalent resistances $r_{f}$ and $r_{p f}$ representing losses. The dc-dc buck converter circuit includes an inductor $L$, a capacitor $C$, equivalent resistances $r_{L}$ and $r_{p}$ standing for losses. The controller aims to regulate all the state variables, i.e., the filter current $i_{f}$, the filter voltage $v_{f}$, the converter current $i_{L}$, and the converter voltage $v_{o}$. The dc-bus voltage is $V_{S}$ and $i_{\text {Load }}$ is the load current. The load can be a CIL, a CCL, or a CPL.

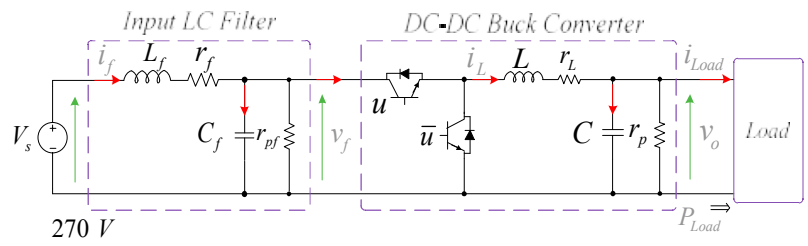

Fig. 4. The topological structure of the studied system.

The dynamic equations of the input $L C$ filter can be described in the following

$$
\begin{gathered}
L_{f} \frac{d i_{f}}{d t}=V_{s}-i_{f} r_{f}-v_{f} \\
C_{f} \frac{d v_{f}}{d t}=i_{f}-\frac{1}{r_{p f}} v_{f}-d i_{L}
\end{gathered}
$$

The dynamic equations of the dc-dc buck converter are written as

$$
\begin{aligned}
& L \frac{d i_{L}}{d t}=d v_{f}-i_{L} r_{L}-v_{o} \\
& C \frac{d v_{o}}{d t}=i_{L}-\frac{v_{o}}{r_{p}}-i_{\text {Load }}
\end{aligned}
$$

where $d$ denotes the duty cycle corresponding to the PWM output signal $u$. Defining the state vector $x=\left[\begin{array}{llll}x_{1} & x_{2} & x_{3} & x_{4}\end{array}\right]^{T}=$ $\left[\begin{array}{llll}i_{f} & v_{f} & i_{L} & v_{o}\end{array}\right]^{T}$ and the energy storage element matrix $Q=$ $\operatorname{diag}\left(L_{f} C_{f} L C\right)$, the total stored energy in the $L C$ filter and the buck converter is expressed by the Hamiltonian function

$$
\begin{aligned}
H(x) & =\frac{1}{2} x^{T} Q x \\
& =\frac{1}{2} L_{f} x_{1}{ }^{2}+\frac{1}{2} C_{f} x_{2}{ }^{2}+\frac{1}{2} L x_{3}{ }^{2}+\frac{1}{2} C x_{4}{ }^{2}
\end{aligned}
$$

In the light of (12), (13) and (14), the dynamics of the studied system can be written in the PCH form, as shown in (15).

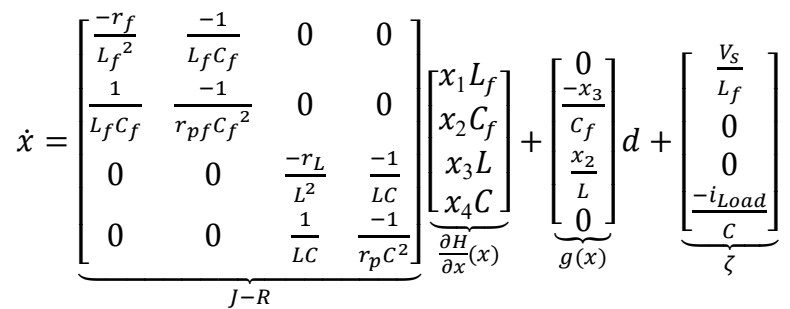

from which matrices $J$ and $R$ can be easily identified:

$$
J=\left[\begin{array}{cccc}
0 & \frac{-1}{L_{f} C_{f}} & 0 & 0 \\
\frac{1}{L_{f} C_{f}} & 0 & 0 & 0 \\
0 & 0 & 0 & \frac{-1}{L C} \\
0 & 0 & \frac{1}{L C} & 0
\end{array}\right] \quad R=\left[\begin{array}{cccc}
\frac{-r_{f}}{L_{f}{ }^{2}} & 0 & 0 & 0 \\
0 & \frac{-1}{r_{p f} C_{f}^{2}} & 0 & 0 \\
0 & 0 & \frac{-r_{L}}{L^{2}} & 0 \\
0 & 0 & 0 & \frac{-1}{r_{p} C^{2}}
\end{array}\right]
$$

Therefore, an overall $\mathrm{PCH}$ model is now ready to design the AESC controller and resolve the stability issue caused by the cascade of filter and converter. However, compared with the PCH model which only considers the dc-dc converter, the order of the proposed model has increased, which may lead to difficulty in solving the system equations. To show this, suppose $x \in R^{n}$ and $u \in R^{p}$. Then, equation (11) can be seen as a set of $n$ equations with $p$ unknowns. In power electronic devices, if $n \neq p$, the solution ( $p$ unknowns satisfying $p<n$ equations) does not exist. As can be seen from (15), $n=4$ and $p=1$, three $(n-p=3)$ new unknowns, considered as three degrees of freedom, should be added to ensure the system has a unique solution. In the following, we will refer to them as $K_{1}$, $K_{2}$, and $K_{3}$.

As mentioned before, the desired interconnection matrix $J_{d}$ and damping matrix $R_{d}$ give a relatively flexible structure for the designer. Indeed, matrix $R_{d}$ needs to remain positive definite to meet the stability requirement. Matrix $J_{d}$ provides a good opportunity to build the internal links in the $\mathrm{PCH}$ structure and increase the degree of freedom. To ensure that the control design admits one unique solution, we introduce variable (timevarying coefficient) $K \in R^{n-p}$ to obtain a non-singular system. In this work, variables $K_{1}, K_{2}, K_{3}$ are introduced in the $\mathrm{PCH}$ system to obtain the unique control law. The desired interconnection and damping matrices in the closed-loop PCH models are defined as follows

$$
\begin{gathered}
J_{d}=J+\left[\begin{array}{cccc}
0 & -K_{1} & 0 & 0 \\
K_{1} & 0 & -K_{2} & 0 \\
0 & K_{2} & 0 & -K_{3} \\
0 & 0 & K_{3} & 0
\end{array}\right] \\
R_{d}=\left[\begin{array}{cccc}
\frac{r_{d 1}}{L_{f}{ }^{2}} & 0 & 0 & 0 \\
0 & \frac{r_{d 2}}{C_{f}{ }^{2}} & 0 & 0 \\
0 & 0 & \frac{r_{d 3}}{L^{2}} & 0 \\
0 & 0 & 0 & \frac{r_{d 4}}{C^{2}}
\end{array}\right]
\end{gathered}
$$


where $r_{d 1}$ to $r_{d 4}$ are to be defined and allow tuning the damping of time responses of the state variables. If no extra damping assigned, i.e. $R_{d}=R, r_{d 1}$ to $r_{d 4}$ correspond to the amount of the circuit in Fig. 4.

The desired operating point $x_{d}$ is the reference of the state variables. It is related to the operating conditions and the circuit characteristic requirements. The buck converter output voltage reference $V_{\text {oref }}$ should be less than $V_{s}=270 \mathrm{~V}$. The desired converter voltage $V_{d}$ and the desired converter current $i_{d}$ are given by

$$
\begin{gathered}
V_{d}=V_{\text {oref }} \\
i_{d}=i_{\text {Load }}+\frac{V_{d}}{r_{p}}
\end{gathered}
$$

Moreover, the desired filter current $i_{f d}$ can be derived by the conservation of the power,

$$
i_{f d}=\frac{V_{s}^{*}}{2 r_{f}^{*}}\left(1-\sqrt{1-\frac{P^{*}}{P_{\max }^{*}}}\right)
$$

where,

$$
\begin{gathered}
V_{s}^{*}=\frac{2 r_{f}+r_{p f}}{r_{p f}} V_{s} \\
r_{f}^{*}=\frac{r_{f}+r_{p f}}{r_{p f}} r_{f} \\
P^{*}=V_{d} i_{\text {Load }}+i_{d}{ }^{2} r_{L}+\frac{V_{s}{ }^{2}}{r_{p f}}+\frac{V_{d}{ }^{2}}{r_{p}} \\
P_{\max }{ }^{*}=\frac{V_{s}^{* 2}}{4 r_{f}{ }^{*}}
\end{gathered}
$$

The desired filter voltage $V_{f d}$ is expressed by

$$
V_{f d}=V_{s}-i_{f d} r_{f}
$$

Compared with other nonlinear control methods, the advantages of the proposed control and other types of PBC are that the method utilizes the physical structure and considers the energy relationship of the system. However, the close connection with the physical structure makes it a model-based control. As can be seen from equations (19) - (22), the parameter variation will influence the desired operating point and thus system performance. The equivalent resistance, i.e., $r_{f}$, $r_{p f}, r_{L}$, and $r_{p}$, can be used to compensate for all the parameter variations. However, a certain set of values will only be satisfied with a given power range. This shortcoming has to be overcome using an on-line parameter observer such as [27] in the future work. It is out of the scope of this paper.
It is worthwhile to note that the proposed control method is not only suitable for several kinds of loads tested in this paper, i.e., CIL, CCL, and CPL, it is also suitable for other types of loads. This is something that the controller in [3] (designed only for CPL) does not have. As can be seen from (22) in [3], the load power $P$ is obtained by multiplying the output voltage and the load current (the product is a constant value). This condition only applies to CPL and does not apply to CIL, CCL, and other types of loads. Because in these cases, the value (calculated by multiplying the output voltage and the load current) is not constant. Thus, the system reference value $x_{d}$ will be a function of the state variable, which makes (10) in this paper no longer applicable. In turn, this invalidates the entire stability proof.

Defining the desired operating point $x_{d}=$ $\left[\begin{array}{llll}x_{1 d} & x_{2 d} & x_{3 d} & x_{4 d}\end{array}\right]^{T}=\left[\begin{array}{llll}i_{f d} & V_{f d} & i_{d} & V_{d}\end{array}\right]^{T}$, the closed-loop Hamiltonian function $H_{d}(x)$ can be described by,

$$
\begin{aligned}
& H_{d}(x)=\frac{1}{2}\left(x-x_{d}\right)^{T} Q\left(x-x_{d}\right) \\
= & \frac{1}{2} L_{f}\left(x_{1}-x_{1 d}\right)^{2}+\frac{1}{2} C_{f}\left(x_{2}-x_{2 d}\right)^{2} \\
+ & \frac{1}{2} L\left(x_{3}-x_{3 d}\right)^{2}+\frac{1}{2} C\left(x_{4}-x_{4 d}\right)^{2}
\end{aligned}
$$

By substituting equations (15), (17), (18) and (23) into equation (11), equation (24) is obtained.

$$
\begin{gathered}
\dot{x}=\left[\begin{array}{cccc}
\frac{-r_{f}}{L_{f}^{2}} & \frac{-1}{L_{f} C_{f}} & 0 & 0 \\
\frac{1}{L_{f} C_{f}} & \frac{-1}{r_{p f} C_{f}^{2}} & 0 & 0 \\
0 & 0 & \frac{-r_{L}}{L^{2}} & \frac{-1}{L C} \\
0 & 0 & \frac{1}{L C} & \frac{-1}{r_{p} C^{2}}
\end{array}\right] \\
\times\left[\begin{array}{cccc}
L_{f} & 0 & 0 & 0 \\
0 & C_{f} & 0 & 0 \\
0 & 0 & L & 0 \\
0 & 0 & 0 & C
\end{array}\right]\left[\begin{array}{l}
x_{1} \\
x_{2} \\
x_{3} \\
x_{4}
\end{array}\right]+\left[\begin{array}{c}
0 \\
\frac{x_{3}}{C_{f}} \\
\frac{x_{2}}{L} \\
0
\end{array}\right] d+\left[\begin{array}{c}
\frac{V_{s}}{L_{f}} \\
0 \\
0 \\
-i_{\text {Load }} \\
C
\end{array}\right]
\end{gathered}
$$

$$
\begin{aligned}
& =\left[\begin{array}{cccc}
\frac{-r_{d 1}}{L_{f}^{2}} & -K_{1}-\frac{1}{L_{f} C_{f}} & 0 & 0 \\
K_{1}+\frac{1}{L_{f} C_{f}} & \frac{-r_{d 2}}{C_{f}^{2}} & -K_{2} & 0 \\
0 & K_{2} & \frac{-r_{d 3}}{L^{2}} & -K_{3}-\frac{1}{L C} \\
0 & 0 & K_{3}+\frac{1}{L C} & \frac{-r_{d 4}}{C^{2}}
\end{array}\right] \\
& \times\left[\begin{array}{cccc}
L_{f} & 0 & 0 & 0 \\
0 & C_{f} & 0 & 0 \\
0 & 0 & L & 0 \\
0 & 0 & 0 & C
\end{array}\right]\left[\begin{array}{l}
x_{1}-x_{1 d} \\
x_{2}-x_{2 d} \\
x_{3}-x_{3 d} \\
x_{4}-x_{4 d}
\end{array}\right]+\left[\begin{array}{l}
\dot{x}_{1 d} \\
\dot{x}_{2 d} \\
\dot{x}_{3 d} \\
\dot{x}_{4 d}
\end{array}\right]
\end{aligned}
$$


This equation is regarded as a set of 4 equations with 4 unknowns. The variables $K_{1}, K_{2}$, and $K_{3}$ are staggered across the rows of (24), establishing internal links in the $\mathrm{PCH}$ framework. Therefore, the existence of the unique solution of the proposed control law is ensured thanks to the fact that these 4 equations are linearly independent.

Moreover, thanks to the variables $K_{1}, K_{2}$, and $K_{3}$, the overall $\mathrm{PCH}$ model is used in the controller design, so all the state variables are regulated by the proposed controller. Therefore, the interactions between the input filter and the dc-dc converter are taken into account in the development of the controller. The large-signal stability is guaranteed by using the overall closedloop Hamiltonian function as Lyapunov function. Furthermore, variables $K_{1}, K_{2}$, and $K_{3}$ are the time-varying coefficient in the control law. They vary with time and operating point. Therefore, an adaptive interconnection matrix $J_{d}$ is also obtained in the proposed AESC.

Variables $K_{1}$ and $K_{3}$ are derived from the first and fourth lines of (24), and they have expressions (25) and (27), respectively. Then, the variable $K_{2}$ can be obtained from the second and third lines of (24), which are shown in (26).

$$
\begin{gathered}
K_{1}=-\frac{1}{L_{f} C_{f}}+\frac{1}{L_{f} C_{f}} K_{1}{ }^{*} \\
K_{2}=\frac{1}{L C_{f}} K_{2}{ }^{*} \\
K_{3}=-\frac{1}{L C}+\frac{1}{L C} K_{3}{ }^{*}
\end{gathered}
$$

The intermediate variables $K_{1}{ }^{*}, K_{2}{ }^{*}$, and $K_{3}{ }^{*}$, which have the following form:

$$
K_{1}{ }^{*}=\frac{r_{f} x_{1}+x_{2}-V_{s}+L_{f} \dot{x}_{1 d}-r_{d 1}\left(x_{1}-x_{1 d}\right)}{x_{2}-x_{2 d}}
$$

$$
K_{3}{ }^{*}=\frac{x_{3}-r_{p}{ }^{-1} x_{4}-i_{\text {Load }}-C \dot{x}_{4 d}+r_{d 4}\left(x_{4}-x_{4 d}\right)}{x_{3}-x_{3 d}}
$$

$K_{2}{ }^{*}=$

$$
\begin{array}{r}
\frac{x_{1} x_{2}-x_{3} x_{4}-r_{p f}{ }^{-1} x_{2}{ }^{2}-r_{L} x_{3}{ }^{2}-C_{f} \dot{x}_{2 d} x_{2}-L \dot{x}_{3 d} x_{3}}{x_{3 d} x_{2}-x_{2 d} x_{3}} \\
+\frac{-K_{1}{ }^{*}\left(x_{1}-x_{1 d}\right) x_{2}+K_{3}{ }^{*}\left(x_{4}-x_{4 d}\right) x_{3}}{x_{3 d} x_{2}-x_{2 d} x_{3}} \\
+\frac{r_{d 2}\left(x_{2}-x_{2 d}\right) x_{2}+r_{d 3}\left(x_{3}-x_{3 d}\right) x_{3}}{x_{3 d} x_{2}-x_{2 d} x_{3}}
\end{array}
$$

Consequently, the final control law $d$ of the proposed AESC is obtained by substituting equations (25)-(30) into the third line of equation (24),

$$
\begin{aligned}
d= & \frac{r_{L} x_{3}+x_{4}+L \dot{x}_{3 d}}{x_{2}} \\
& +\frac{K_{2}{ }^{*}\left(x_{2}-x_{2 d}\right)-r_{d 3}\left(x_{3}-x_{3 d}\right)-K_{3}{ }^{*}\left(x_{4}-x_{4 d}\right)}{x_{2}}
\end{aligned}
$$

The overall control scheme with the proposed AESC controller is shown in Fig. 5. As shown, a more detailed model of the input $L C$ filter and dc-dc buck converter is used. Some parasitic elements and their influences on the stability and control are considered. In this work. all the dynamics of the input filter and buck converter are included in the proposed control algorithm. Therefore, the risk of instability caused by the interactions between the filter and the converter is taken into account during the controller design. Moreover, three different loads, i.e., CIL, CCL, and CPL, are considered and tested to connect with the studied converter system. The controller design also takes into account different load conditions. The simulation and experimental results under these three load conditions are given in the following two sections, and the results are also analyzed and compared. Furthermore, the proposed method is also compared with the conventional IDAPBC and the conventional PI control.

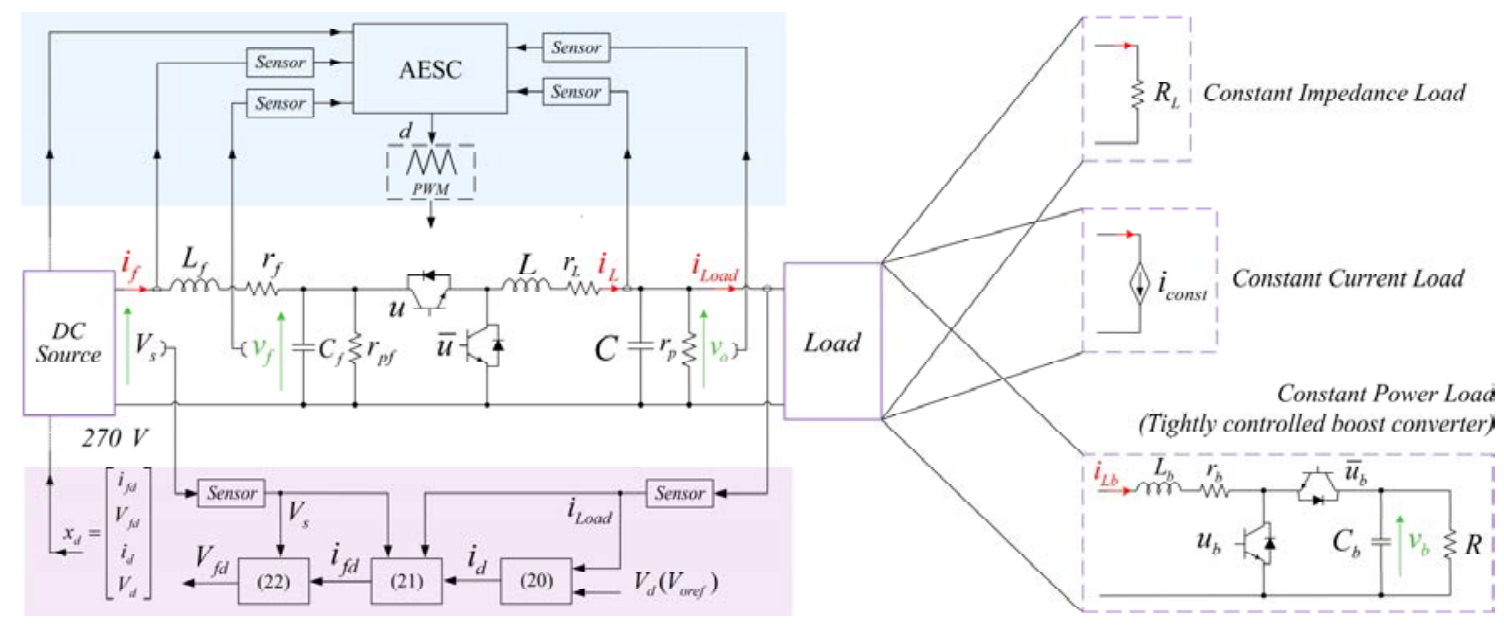

Fig. 5. The overall control algorithm of the studied system. 
The method of selecting damping introduced in Section III is a general guideline for damping selection. As for the converter system developed in this work, the damping matrix $R_{d}$ consists of four damping factors, i.e. $r_{d 1}, r_{d 2}, r_{d 3}$, and $r_{d 4}$. Indeed, in power electronic case, dissipation always exists and is positive $(R>0)$, so natural damping $\left(R_{d}=R\right)$ is sufficient to ensure system stability. This can actually be verified by simulation and experimental results given in [25] and also in this paper. Since the natural damping usually cannot meet the dynamic requirements, one can choose any number of damping factors in $R_{d}$ to inject damping. However, on the one hand, too much damping will cause the duty cycle to reach saturation during the transient, which will invalidate the system's stability proof, thereby increasing the risk of system instability. On the other hand, although too much damping can significantly reduce overshoot/undershoot, it can also slow down the system response, resulting in a longer settling time. Here, the strategy is to find an effective damping factor in $R_{d}$ to inject the virtual damping and let other damping factors keep their original values. This facilitates damping injection, and is easy to monitor and ensure that the duty cycle will not reach saturation. As can be seen in Eq. (31), $r_{d 3}$ is directly related to the final control law. The damping factor $r_{d 2}$ in $K_{2}{ }^{*}$ of Eq. (30), $r_{d 4}$ in $K_{3}{ }^{*}$ of Eq. (29), and $r_{d 1}$ in $K_{1}{ }^{*}$ of Eq. (28) are indirectly related to the final control law. Therefore, $r_{d 3}$ is more closely related to the dynamic characteristics, and the required damping will be set more effectively. This has also been verified by comparing the simulation results between $r_{d 1}, r_{d 2}, r_{d 3}$, and $r_{d 4}$. Therefore, we present the system responses for different values of $r_{d 3}$ in the paper.

\section{Simulation Results}

To verify the proposed control algorithm, the simulation tests are carried out in Matlab/Simulink. The validation of the control law is performed under different types of load variations, which represent three scenarios that are of interest in practical applications.

TABLE I

System Parameters

\begin{tabular}{ll}
\hline \hline \multicolumn{1}{c}{ Item } & Value \\
\hline Input Voltage $V_{s}$ & $270 \mathrm{~V}$ \\
Output Voltage Reference $V_{\text {oref }}$ & $200 \mathrm{~V}$ \\
Filter Inductance $L_{f}$ & $246 \mu \mathrm{H}$ \\
Filter Equivalent Series Resistance $r_{f}$ & $0.05 \Omega$ \\
Filter Capacitance $C_{f}$ & $200 \mu \mathrm{F}$ \\
Filter Equivalent Parallel Resistance $r_{p f}$ & $10 \mathrm{M} \Omega$ \\
Buck Inductance $L$ & $950 \mu \mathrm{H}$ \\
Buck Equivalent Series Resistance $r_{L}$ & $0.2 \Omega$ \\
Buck Capacitance $C$ & $420 \mu \mathrm{F}$ \\
Buck Equivalent Parallel Resistance $r_{p}$ & $5 \mathrm{M} \Omega$ \\
Frequency $f_{s}$ & $20 \mathrm{kHz}$ \\
\hline \hline
\end{tabular}

The parameters used in this paper are given in Table I. In practice, designers can set unique control objectives according to actual needs and different applications. When the damping is injected into the system, several control indicators (settling time, overshoot/undershoot of current or voltage, etc.) will change, shorter settling time is always of interest in practical applications. Here, the control objective we set is to reduce the settling time to less than $5 \mathrm{~ms}$. This objective can conveniently help us compare the results of no damping injection case with damping injection case. The state variable $x=\left[\begin{array}{llll}i_{f} & v_{f} & i_{L} & v_{o}\end{array}\right]^{T}$ and the desired operating point $x_{d}=\left[i_{f d} V_{f d} i_{d} V_{d}\right]^{T}$ are depicted in the same figure to illustrate the effective tracking performance of the proposed AESC approach.

\section{A. Scenario 1: Sudden CIL Increment}

In this case study, the CIL is connected to the system and the resistance is stepped from $54 \Omega$ to $16 \Omega$ at $t=0.13 \mathrm{~s}$. To this end, the load power changes from $0.7 \mathrm{~kW}$ to $2.5 \mathrm{~kW}$. Initially, the damping is not injected into the system $\left(R_{d}=R\right)$. The damping factors $r_{d 1}, r_{d 2}, r_{d 3}$, and $r_{d 4}$ in $R_{d}$ are selected to be equal to their nominal values, whose values are the parameters defined in Table I. As can be seen from Fig. 6, all the state variables can accurately track their desired value, and the settling time is around $20 \mathrm{~ms}$ in this case. In contrast, if the damping is injected into the system (see Fig. 7), the settling time can be reduced to $5 \mathrm{~ms}$ when $r_{d 3}=1.7$. The value of the damping factor is chosen by trial and error. Compared with the no damping case, the peak overshoot of the inductor current is reduced by $50 \%$, and the settling time is reduced by $75 \%$.
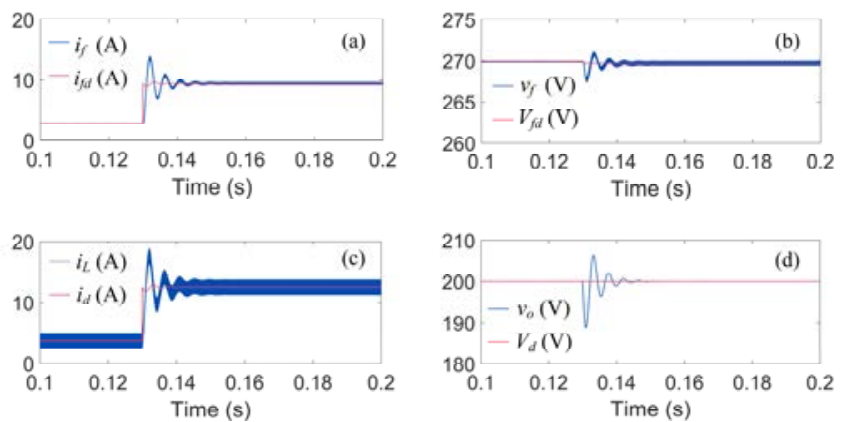

Fig. 6. The simulation waveforms without damping injection when CIL steps from $54 \Omega$ to $16 \Omega$. (a) Filter current. (b) Filter voltage. (c) Converter current. (d) Converter voltage.
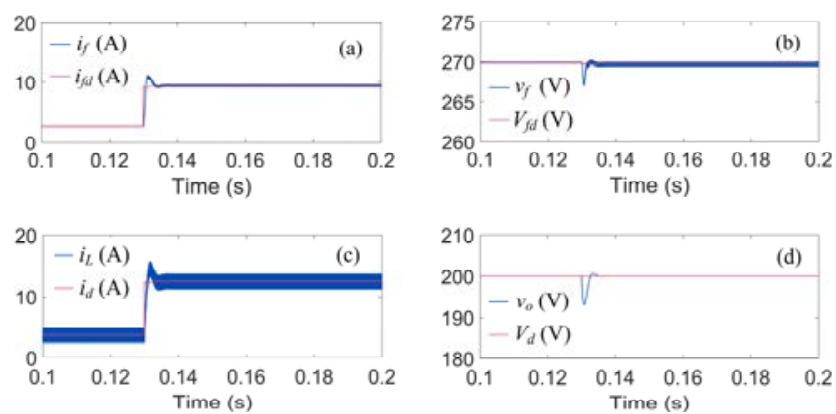

Fig. 7. The simulation waveforms with damping injection when CIL steps from $54 \Omega$ to $16 \Omega$. (a) Filter current. (b) Filter voltage. (c) Converter current. (d) Converter voltage.

\section{B. Scenario 2: Sudden CCL Increment}

This scenario has been implemented to demonstrate the applicability of the proposed AESC technique to variable CCL. The load current steps from $5 \mathrm{~A}$ to $12.5 \mathrm{~A}$, which corresponds to the load power steps from $1 \mathrm{~kW}$ to $2.5 \mathrm{~kW}$. Fig. 8 shows the 
simulation waveforms without damping injection when the load varies. The settling time is about $30 \mathrm{~ms}$ in both current and voltage. The same simulation is done for the enabled damping injection. As shown in Fig. 9, when $r_{d 3}$ is set to 1.9, the system takes about $5 \mathrm{~ms}$ to reach its new operating point. Compared to the results given in Fig. 8, the peak overshoot of the inductor current is reduced by $48.1 \%$, and the settling time is reduced by $83.3 \%$.
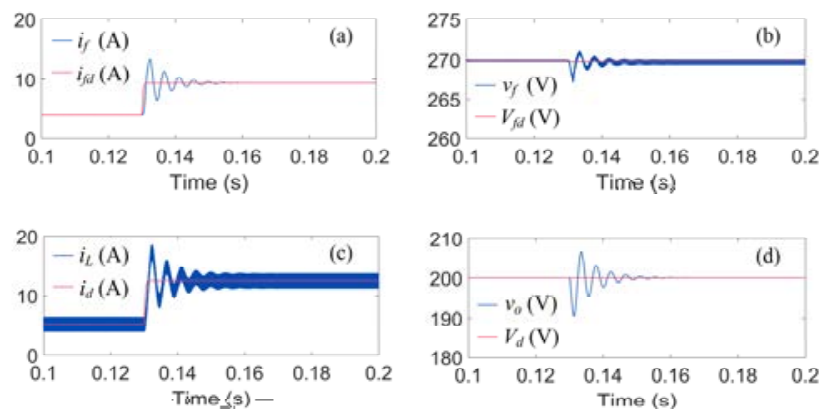

Fig. 8. The simulation waveforms without damping injection when CCL steps from 5 A to 12.5 A. (a) Filter current. (b) Filter voltage. (c) Converter current. (d) Converter voltage.
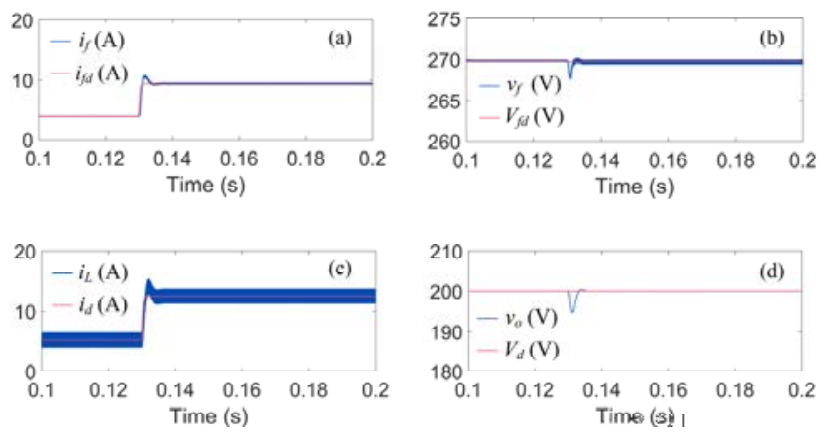

Fig. 9. The simulation waveforms with damping injection when CCL steps from 5 A to 12.5 A. (a) Filter current. (b) Filter voltage. (c) Converter current. (d) Converter voltage.

\section{Scenario 3: Sudden CPL Increment}

As mentioned before, the CPL is the most critical situation in terms of stability. The objective of this simulation study is to verify the feasibility of stable operation under the CPL. The simulation verification with CPL follows the same steps outlined above for CIL and CCL.
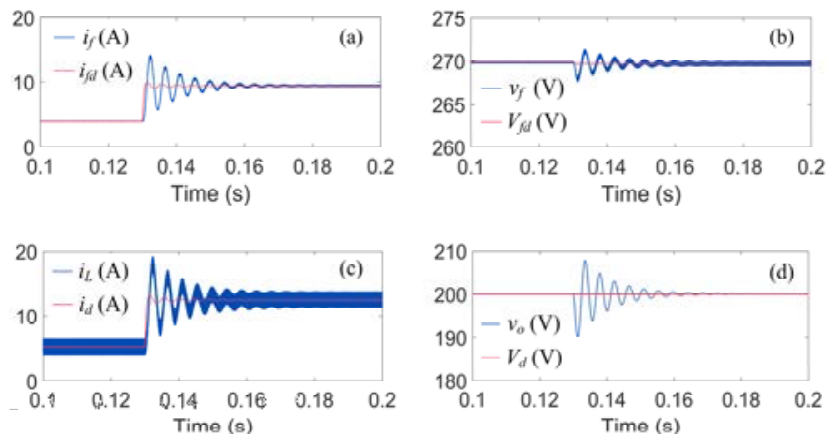

Fig. 10. The simulation waveforms without damping injection when CPL steps from $1 \mathrm{~kW}$ to $2.5 \mathrm{~kW}$. (a) Filter current. (b) Filter voltage. (c) Converter current. (d) Converter voltage.
Firstly, the damping is not injected into the system. As can be seen from Fig. 10, a relatively large overshoot and long settling time are responded to this kind of step. The settling time is around $50 \mathrm{~ms}$. Afterward, the damping is injected into the system $\left(r_{d 3}=2.2\right)$. As shown in Fig. 11, the dynamic is tuned to show an improved performance during the transient process. Compared to the results given in Fig. 10, the inductor current overshoot is reduced by $48.3 \%$, and the settling time is reduced by $90 \%$.
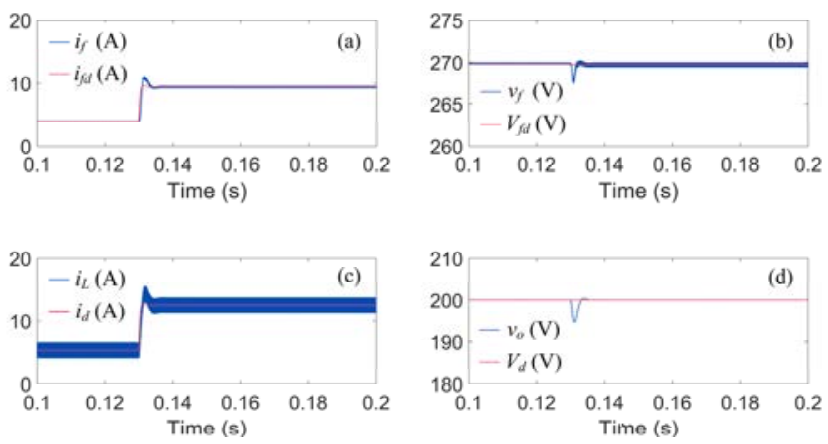

Fig. 11. The simulation waveforms with damping injection when CPL steps from $1 \mathrm{~kW}$ to $2.5 \mathrm{~kW}$. (a) Filter current. (b) Filter voltage. (c) Converter current. (d) Converter voltage.

Moreover, the proposed control strategy is compared with conventional IDA-PBC and well-tuned PI control. Consider that all three control strategies work in a power range where everyone is stable. The CPL load steps from $1 \mathrm{~kW}$ to $2 \mathrm{~kW}$. The simulation results are shown in Fig. 12, Fig. 13, and Fig. 14, respectively.
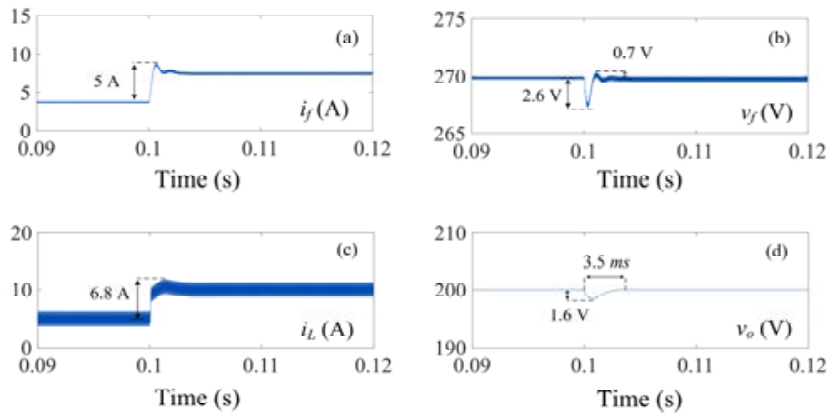

Fig. 12. Simulation results of the proposed AESC. (a) Filter current. (b) Filter voltage. (c) Converter current. (d) Converter voltage.
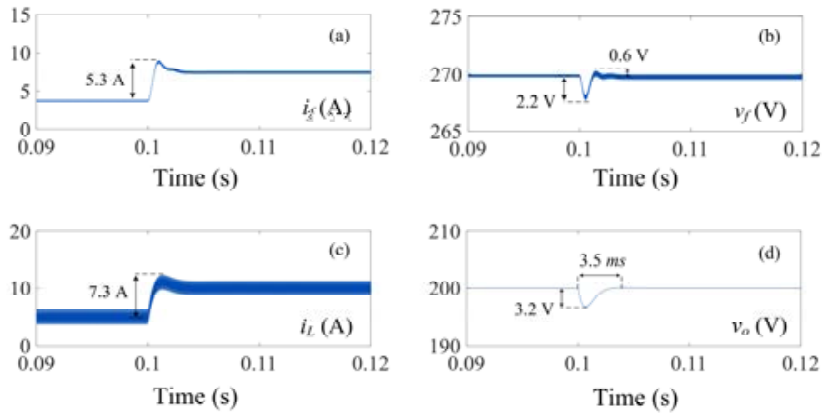

Fig. 13. Simulation results of the conventional IDA-PBC. (a) Filter current. (b) Filter voltage. (c) Converter current. (d) Converter voltage. 
TABLE II

The Dynamic Performance Comparison of Three Control Strategies

\begin{tabular}{cccc}
\hline \hline Control Algorithm & $\begin{array}{l}\text { Converter Inductor } \\
\text { Current Overshoot }\end{array}$ & $\begin{array}{l}\text { The Improvement } \\
\text { (Compared to PI) }\end{array}$ & $\begin{array}{l}\text { Converter Capacitor } \\
\text { Voltage Undershoot }\end{array}$ \\
\hline Proposed AESC & $18 \%$ & $37.9 \%$ & $-0.8 \%$ \\
(Compared to PI) & $-1.6 \%$ & $61.9 \%$ \\
Conventional IDA-PBC & $23 \%$ & $20.7 \%$ & $-2.1 \%$ \\
Conventional PI Control & $29 \%$ & -- & -- \\
\hline \hline
\end{tabular}
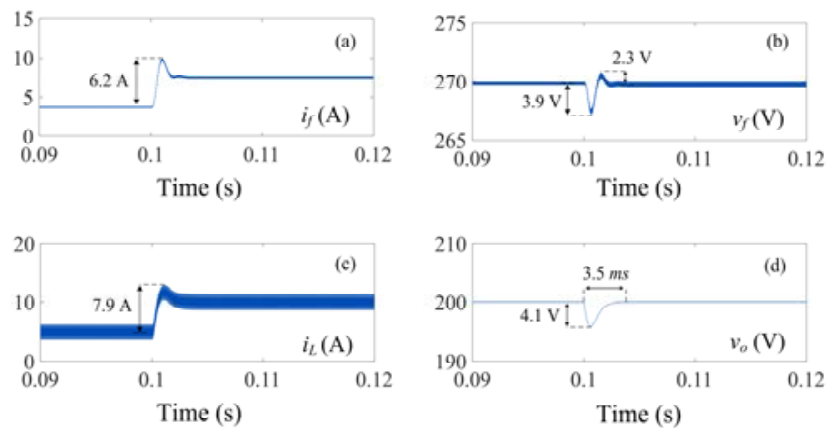

Fig. 14. Simulation results of the PI control. (a) Filter current. (b) Filter voltage. (c) Converter current. (d) Converter voltage.

The damping parameter used for AESC is the same as Fig. 11. Here, we tune these control strategies to have the same settling time and compare the overshoot/undershoot of the systems. The results are given in Table II. In general, the proposed AESC has stronger robustness than the other two control strategies. The conventional IDA-PBC has better performance than the welltuned PI control.

\section{EXPERIMENTAL RESULTS}

To validate the effectiveness of the proposed AESC control approach, a $270 \mathrm{~V}-3.5 \mathrm{~kW}$ experiment setup was built which is shown in Fig. 15. The control algorithm is conducted based on MATLAB/Simulink and then coded into dSPACE (ds1103) real-time control card.

Three scenarios have also been implemented to illustrate the applicability of the proposed control algorithm to various loads with different characteristics. The parameter used is the same as in Table I.

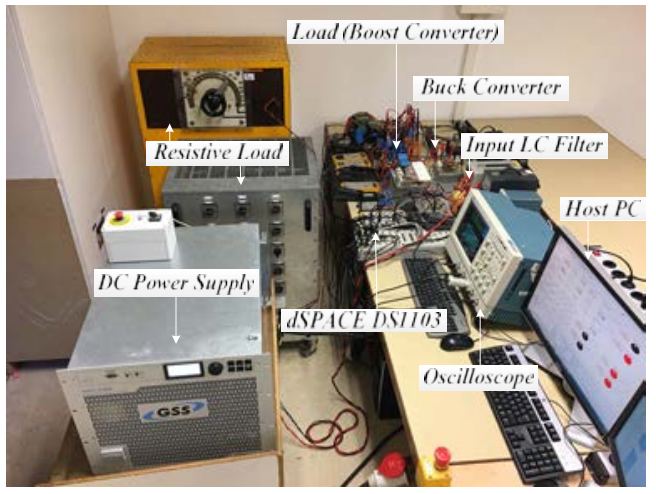

Fig. 15. $270 \mathrm{~V}-3.5 \mathrm{~kW}$ experiment setup.

This paper not only verifies the feasibility and effectiveness of the proposed method by using dSPACE real-time control card, but also verifies the possibility of applying the proposed method on a regular embedded processor. Here, a TI-DSP, i.e., TMS320F28335 (Fig. 16), is also used to verify the possibility of the control algorithm running on a regular embedded processor, and compare the execution time of the proposed control algorithm with the execution time of conventional IDA$\mathrm{PBC}$ and conventional PI control. It is worthwhile to note that, this DSP card is frequently used, but it is rather an old member of the TI-DSP family. The results are shown in Table III.

Through the Code Composer Studio (CCS) integrated development environment, the execution time of the proposed AESC algorithm is $12.91 \mu$ s. Thus, this control law is feasible to use for industrial applications. Compared with the conventional IDA-PBC [25], the complexity of the proposed control law has not significantly increased. Of course, this is much more than what a PI controller needs, but commercial digital controllers like TI-DSPs can easily cope with it.

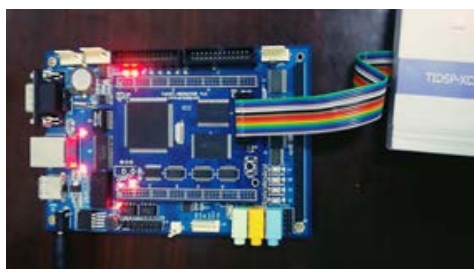

Fig. 16. TI-DSP: TMS320F28335.

TABLE III

The Execution Time Comparison with TMS320F28335

\begin{tabular}{lc}
\hline \hline Control Algorithm & Execution Time \\
\hline Proposed AESC & $12.91 \mu \mathrm{s}$ \\
Conventional IDA-PBC & $3.86 \mu \mathrm{s}$ \\
Conventional PI Control & $0.95 \mu \mathrm{s}$ \\
\hline \hline
\end{tabular}

\section{A. Scenario 1: Sudden CIL Change}

The first experiment is concerned with the studied system supplying CIL, a resistive load is used in this scenario. Initially, the load resistance is changed from $54 \Omega$ to $16 \Omega$. Next, the resistance is changed from $11.5 \Omega$ to $25 \Omega$. It is worthwhile to mention that the resistance value may fluctuate in practice due to the environment and operating temperature.

It is clearly observed from Figs. 17-20 that all the state variables settle to their desired values under the two cases. In Fig. 17, the damping is not injected into the system when the resistive load changes from $54 \Omega$ to $16 \Omega$, i.e. the load power changes from $0.7 \mathrm{~kW}$ to $2.5 \mathrm{~kW}$. This change can be considered a more severe situation compared to the case from $40 \Omega$ to 16 $\Omega(1 \mathrm{~kW}$ to $2.5 \mathrm{~kW})$. The results show accurate trajectory tracking without instability issues. However, there are a relatively long settling time and a large overshoot/undershoot during the transient. Fig. 18 presents the results when the damping factor $r_{d 3}$ is set to 1.7 (choose according to simulation), both settling time and overshoot/undershoot are reduced thanks to the damping injection technique. 
TABLE IV

The Dynamic Performance Comparison of CIL

\begin{tabular}{|c|c|c|c|c|c|c|}
\hline & \multicolumn{3}{|c|}{$54 \Omega \rightarrow 16 \Omega$} & \multicolumn{3}{|c|}{$11.5 \Omega \rightarrow 25 \Omega$} \\
\hline & Converter Inductor & Converter Capacitor & Settling & Converter Inductor & Converter Capacitor & Settling \\
\hline & Current Overshoot & Voltage Undershoot & Time & Current Undershoot & Voltage Overshoot & Time \\
\hline Without Damping Injection & $53.9 \%$ & $-5.5 \%$ & $15 \mathrm{~ms}$ & $-64.7 \%$ & $2.5 \%$ & $14 \mathrm{~ms}$ \\
\hline With Damping Injection & $26.9 \%$ & $-3 \%$ & $5 \mathrm{~ms}$ & $-35.3 \%$ & $1.5 \%$ & $4 \mathrm{~ms}$ \\
\hline The Improvement & $50.1 \%$ & $45.5 \%$ & $66.7 \%$ & $45.4 \%$ & $40 \%$ & $71.4 \%$ \\
\hline
\end{tabular}

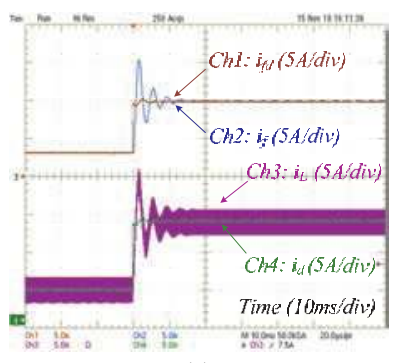

(a)

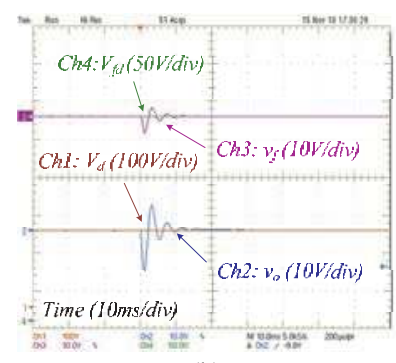

(b)

Fig. 17. The experimental waveforms without damping injection when CIL steps from $54 \Omega$ to $16 \Omega$. (a) Current. (b) Voltage.

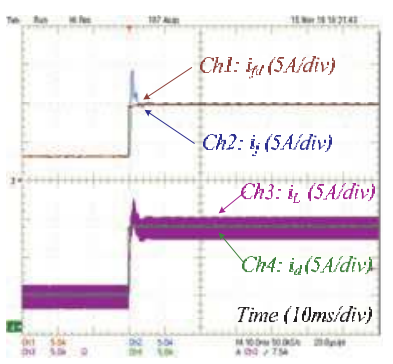

(a)

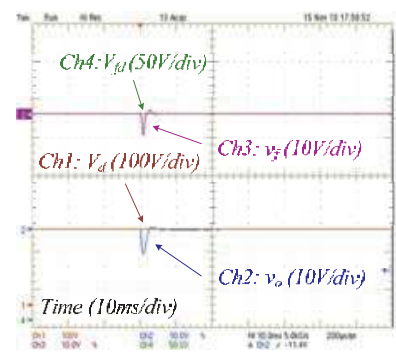

(b)

Fig. 18. The experimental waveforms with damping injection when CIL steps from $54 \Omega$ to $16 \Omega$. (a) Current. (b) Voltage.

Moreover, Figs. 19 and 20 illustrate the system response when the resistive load changes from $11.5 \Omega$ to $25 \Omega$, i.e. the load power changes from $3.5 \mathrm{~kW}$ to $1.6 \mathrm{~kW}$. Their damping factor values are the same as in Fig. 17 and Fig. 18, respectively. A good trajectory tracking performance in current and voltage are also verified.

Table IV summarizes and shows the numerical comparison of the results embedded in Figs. 17-20. As can be seen from the table, the damping injection technique can indeed improve the transient performance of the system.

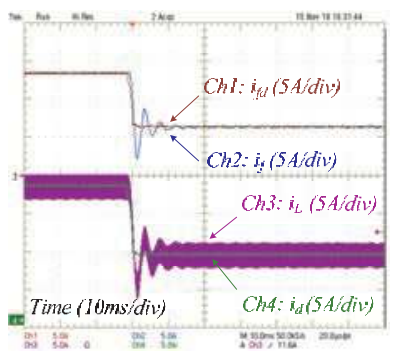

(a)

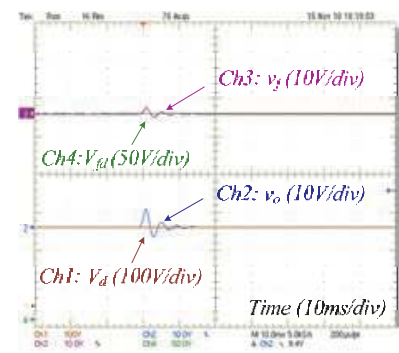

(b)

Fig. 19. The experimental waveforms without damping injection when CIL steps from $11.5 \Omega$ to $25 \Omega$. (a) Current. (b) Voltage.

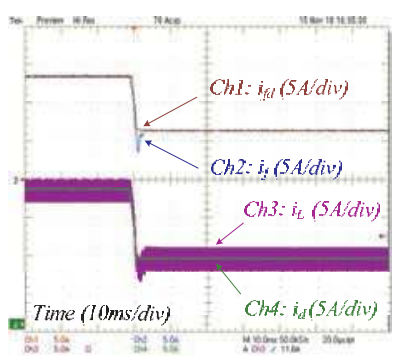

(a)

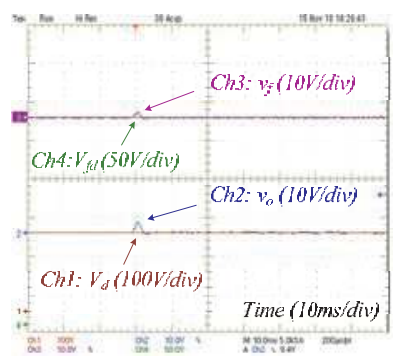

(b)
Fig. 20. The experimental waveforms with damping injection when CIL steps from $11.5 \Omega$ to $25 \Omega$. (a) Current. (b) Voltage.

\section{B. Scenario 2: Sudden CCL Change}

For the second scenario, the load current of the CCL is initially set to $5 \mathrm{~A}$ and then increased to $12.5 \mathrm{~A}$, i.e. the load power changes from $1 \mathrm{~kW}$ to $2.5 \mathrm{~kW}$. Their damping factor values are the same as those used in the simulation. It can be clearly seen from Figs. 21 and 22, all the state variables track desired values well under step change in the CCL current.

Table V compares the experimental results based on Fig. 21 and Fig. 22. And again, seen from the table, comparing the no damping case, i.e. Fig. 21 with enabled damping case, i.e. Fig. 22 , the transient performance is improved due to the damping injection.

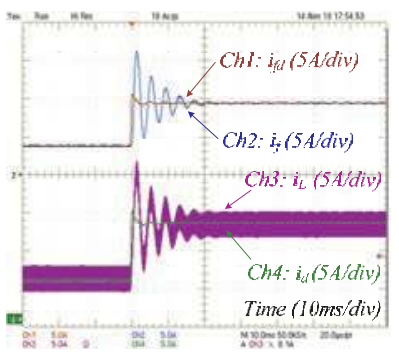

(a)

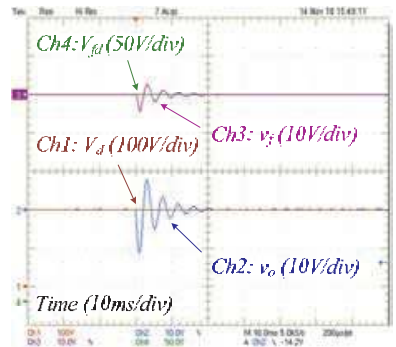

(b)
Fig. 21. The experimental waveforms without damping injection when CCL steps from 5 A to 12.5 A. (a) Current. (b) Voltage.

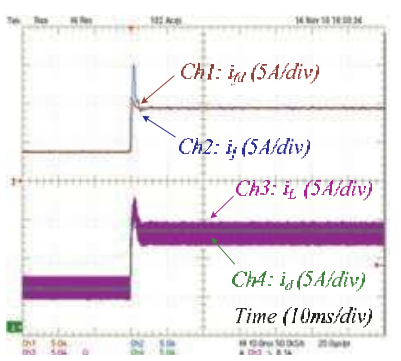

(a)

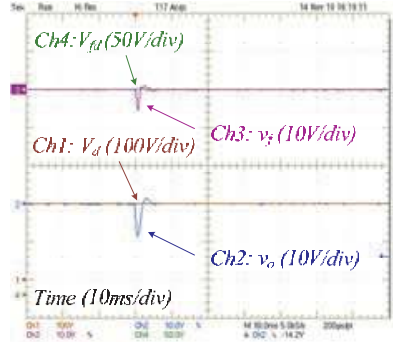

(b)
Fig. 22. The experimental waveforms with damping injection when CCL steps from 5 A to 12.5 A. (a) Current. (b) Voltage. 
TABLE V

The Dynamic Performance Comparison of CCL

\begin{tabular}{lccc}
\hline \hline & & $5 \mathrm{~A} \rightarrow 12.5 \mathrm{~A}$ & \\
\hline & Converter Inductor & Converter Capacitor & Settling \\
& Current Overshoot & Voltage Undershoot & Time \\
Without Damping Injection & $68 \%$ & $-5.5 \%$ & $24 \mathrm{~ms}$ \\
With Damping Injection & $36 \%$ & $-4 \%$ & $6 \mathrm{~ms}$ \\
The Improvement & $47.1 \%$ & $27.3 \%$ & $75 \%$ \\
\hline \hline
\end{tabular}

\section{Scenario 3: Sudden CPL Change}

The third experiment is carried out to examine the proposed control method under CPL in the presence of the step change of load power. It is worthwhile to note that, the ideal CPL does not exist in reality. The tightly regulated converter load can behave like CPL. Thus, it is equitable to adopt a tightly regulated dc-dc converter as the CPL. In this work, CPL is achieved by developing a tightly regulated boost converter that supplies a resistive load (see Fig. 5).

For the first case, the load power of the CPL steps from $1 \mathrm{~kW}$ to $2.5 \mathrm{~kW}$. Fig. 23 shows the system performance when the damping is not injected into the system. Afterward, the damping is injected into the system. Fig. 24 and Fig .25 illustrate the results for the cases $r_{d 3}=0.5$ and $r_{d 3}=2.2$, respectively. As shown, all the state variables can track their desired values and the damping injection technique can improve the transient performance.

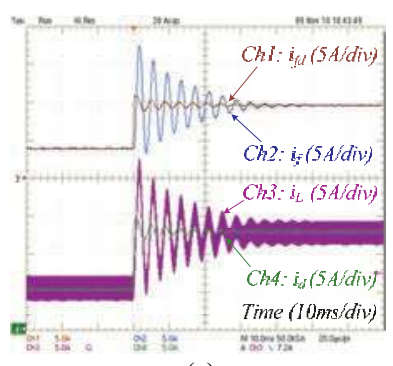

(a)

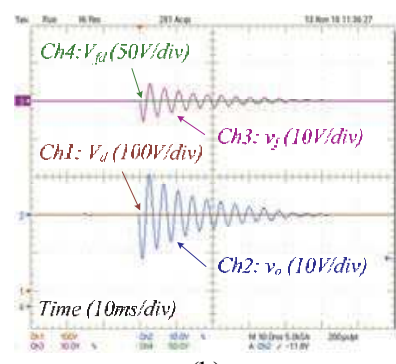

(b)
Fig. 23. The experimental waveforms without damping injection when CPL steps from $1 \mathrm{~kW}$ to $2.5 \mathrm{~kW}$. (a) Current. (b) Voltage.

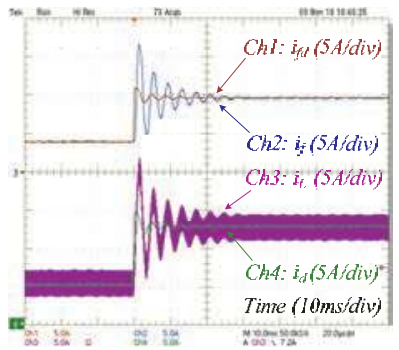

(a)

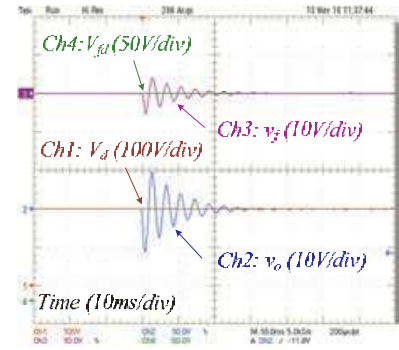

(b)
Fig. 24. The experimental waveforms with $r_{d 3}=0.5$ when CPL steps from 1 $\mathrm{kW}$ to $2.5 \mathrm{~kW}$.

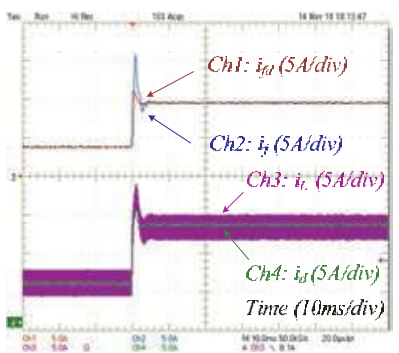

(a)

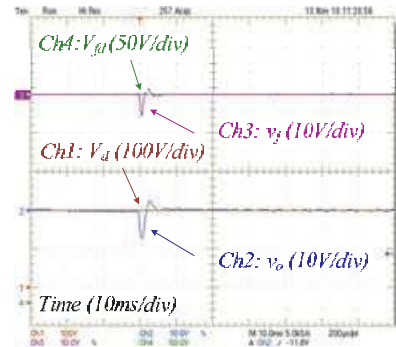

(b)
Fig. 25. The experimental waveforms with $r_{d 3}=2.2$ when CPL steps from 1 $\mathrm{kW}$ to $2.5 \mathrm{~kW}$. (a) Current. (b) Voltage.
For the second case, the load power of the CPL steps from $3.5 \mathrm{~kW}$ to $2 \mathrm{~kW}$. The results are shown in Figs 26-28. As observed, the system behaves as desired with the state variables closely tracking the reference values. Table VI summarizes and shows the numerical comparison of the results embedded in Figs 23-28.

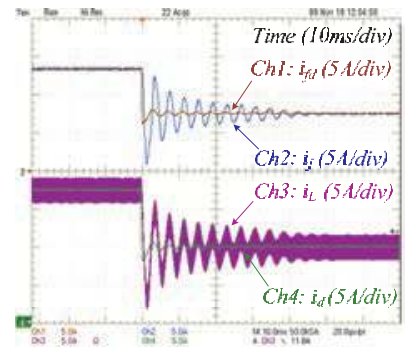

(a)

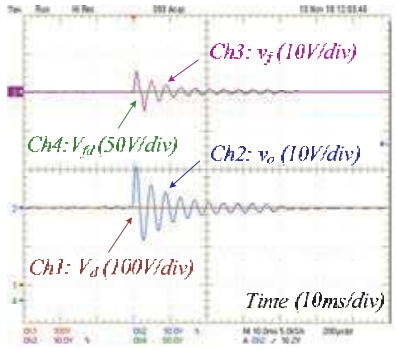

(b)
Fig. 26. The experimental waveforms without damping injection when CPL steps from $3.5 \mathrm{~kW}$ to $2 \mathrm{~kW}$. (a) Current. (b) Voltage.

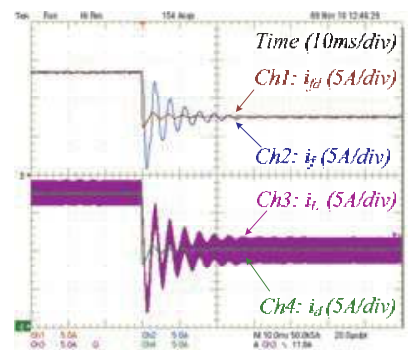

(a)

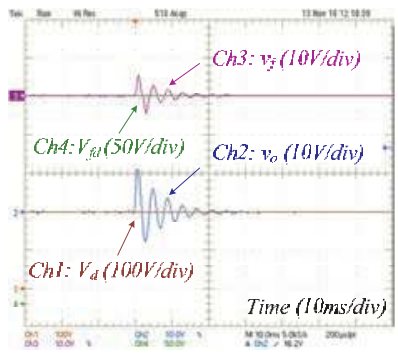

(b)
Fig. 27. The experimental waveforms with $r_{d 3}=0.5$ when CPL steps from 3.5 $\mathrm{kW}$ to $2 \mathrm{~kW}$. (a) Current. (b) Voltage.

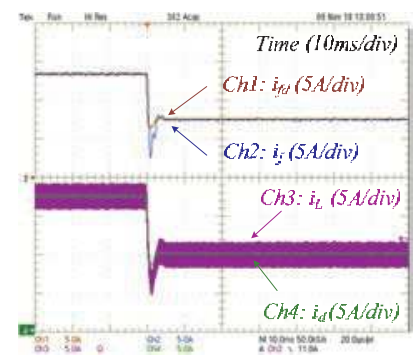

(a)

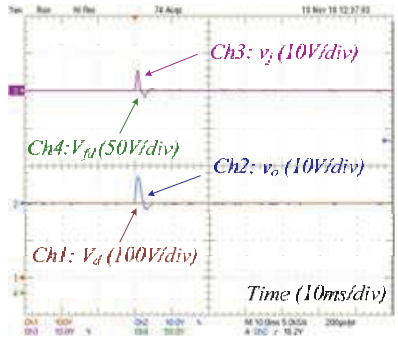

(b)
Fig. 28. The experimental waveforms with $r_{d 3}=2.2$ when CPL steps from 3.5 $\mathrm{kW}$ to $2 \mathrm{~kW}$. (a) Current. (b) Voltage.

Based on all the above verification results, the proposed control approach successfully maintains all the state variables at the desired value regardless of variations of the different types of loads. The dynamic performance comparison between simulation and experiment can be summarized in Table VII. As shown, the damping injection technique in all cases can ensure the system has good dynamic performance during the transient. In Table VII, the indicators of simulation and experimental results are not exactly the same, which may be caused by the following reasons:

1) Model error is the main reason for this difference. Even if dissipative resistances, i.e., $r_{f}, r_{p f}, r_{L}$, and $r_{p}$, can be equivalent to some un-modeled losses and compensate for the steady-state error, it cannot accurately describe the actual system and completely characterize the dynamic performance of the system. 
TABLE VI

The Dynamic Performance Comparison of CPL

\begin{tabular}{|c|c|c|c|c|c|c|}
\hline & \multicolumn{3}{|c|}{$1 \mathrm{~kW} \rightarrow 2.5 \mathrm{~kW}$} & \multicolumn{3}{|c|}{$3.5 \mathrm{~kW} \rightarrow 2 \mathrm{~kW}$} \\
\hline & Converter Inductor & Converter Capacitor & Settling & Converter Inductor & Converter Capacitor & Settling \\
\hline & Current Overshoot & Voltage Undershoot & Time & Current Undershoot & Voltage Overshoot & Time \\
\hline Without Damping Injection & $80 \%$ & $-6 \%$ & $50 \mathrm{~ms}$ & $-80 \%$ & $5.3 \%$ & $50 \mathrm{~ms}$ \\
\hline With Damping Injection $\left(r_{d 3}=0.5\right)$ & $76 \%$ & $-5.5 \%$ & $30 \mathrm{~ms}$ & $-80 \%$ & $5 \%$ & $30 \mathrm{~ms}$ \\
\hline With Damping Injection $\left(r_{d 3}=2.2\right)$ & $40 \%$ & $-4 \%$ & $6 \mathrm{~ms}$ & $-50 \%$ & $3.5 \%$ & $6 \mathrm{~ms}$ \\
\hline The Improvement * & $50 \%$ & $33.3 \%$ & $88 \%$ & $37.5 \%$ & $40 \%$ & $88 \%$ \\
\hline
\end{tabular}

* The improvement is to compare the no damping case with the case of $r_{d 3}=2.2$.

TABLE VII

The Dynamic Performance Comparison Between Simulation and Experiment

\begin{tabular}{|c|c|c|c|c|c|c|c|c|}
\hline & \multicolumn{4}{|c|}{ Simulation } & \multicolumn{4}{|c|}{ Experiment } \\
\hline & \multicolumn{2}{|c|}{ Without Damping Injection } & \multicolumn{2}{|c|}{ With Damping Injection } & \multicolumn{2}{|c|}{ Without Damping Injection } & \multicolumn{2}{|c|}{ With Damping Injection } \\
\hline & Converter Inductor & Settling & Converter Inductor & Settling & Converter Inductor & Settling & Converter Inductor & Settling \\
\hline & Current Overshoot & Time & Current Overshoot & Time & Current Overshoot & Time & Current Overshoot & Time \\
\hline $\mathrm{CIL}^{*}$ & $52 \%$ & $20 \mathrm{~ms}$ & $26 \%$ & $5 \mathrm{~ms}$ & $54 \%$ & $15 \mathrm{~ms}$ & $27 \%$ & $5 \mathrm{~ms}$ \\
\hline $\mathrm{CCL}^{* *}$ & $52 \%$ & $30 \mathrm{~ms}$ & $27 \%$ & $5 \mathrm{~ms}$ & $68 \%$ & $24 \mathrm{~ms}$ & $36 \%$ & $6 \mathrm{~ms}$ \\
\hline $\mathrm{CPL}^{* * *}$ & $60 \%$ & $50 \mathrm{~ms}$ & $31 \%$ & $5 \mathrm{~ms}$ & $80 \%$ & $50 \mathrm{~ms}$ & $40 \%$ & $6 \mathrm{~ms}$ \\
\hline
\end{tabular}

* CIL steps from $54 \Omega$ to $16 \Omega$, damping injection with $r_{d 3}=1.7$. $* *$ CCL steps from 5 A to 12.5 A, damping injection with $r_{d 3}=1.9$.

*** CPL steps from $1 \mathrm{~kW}$ to $2.5 \mathrm{~kW}$, damping injection with $r_{d 3}=2.2$.

2) In practice, various external and internal noises will affect the system performance, especially transient performance.

3) As for the CIL, an adjustable resistive load is used, this resistance may vary with the environment, especially the temperature. Besides, manually operating the switch (changing the resistance value) could cause delay, and the arc generated by the operating switch will also affect the transient performance, so this imperfect resistive load cannot completely simulate the same resistance step as in the simulation.

4) Regarding the CCL, a dc-dc converter with a controller that makes the converter work in the constant current mode is first used as CCL, and then an electronic load that works in the constant current mode is also tested. The results are basically the same. However, both of these are different from the ideal $\mathrm{CCL}$ in the simulation.

5) About the CPL, as mentioned before, a tightly regulated boost converter (with nonlinear controller) is used to emulate the CPL. Due to its bandwidth limitation, the electronic load is not used here. The CPL characteristic of the electronic load can only be ensured in steady-state. In transients, it cannot be expressed as CPL characteristics. Overall, the result of this equivalent $\mathrm{CPL}$ is naturally different from the result of the ideal CPL in the simulation.

\section{CONCLUSION}

In this paper, the stability issue of the cascade configuration of the dc-dc power converter and the input filter has been addressed. Such a system appears frequently in on-board dc microgrids and can be considered as a typical subsystem of the microgrid system. The stabilization is achieved by developing an AESC based on the PCH framework.

It is shown that the input filter affects the stability of the system. To solve this issue, an overall PCH model including an adaptive interconnection matrix is built to ensure the interactions between the filter and the converter is regulated by the proposed control approach. Moreover, to tackle the instability potential when several subsystems are put in cascade in a microgrid, the proposed AESC scheme also considers the interactions between several subsystems.

In addition to these theoretical backgrounds, the simulation and experimentation under different load conditions, i.e., CIL, CCL, and CPL, are also carried out to demonstrate the effectiveness of the proposed control law. Compared to the conventional IDA-PBC (Fig. 3), the proposed method can not only ensure the large-signal stability of the power converter cascaded with its input filter, but also ensure the stability when cascading multiple subsystems. Besides, the comparison results also show that the dynamic performance of the proposed method is better than the conventional IDA-PBC and the welltuned PI control.

\section{APPENDIX \\ -DESIGN INPUT FILTER FOR BUCK CONVERTER}

Considering the topology given in Fig. A1, an input filter is added to the power input terminal of the dc-dc buck converter.

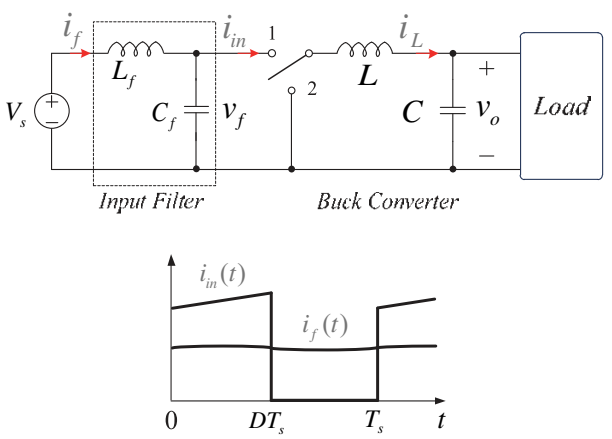

Fig. A1. Addition of an input filter to the power input terminal of the buck converter: (a) Circuit. (b) Input current waveforms. 
According to Fig. A1, the input current of the buck converter $i_{\text {in }}(t)$ in steady-state has the following expression:

$$
i_{\text {in }}(t)=\left\{\begin{array}{cl}
I_{L}-\frac{1}{2} \Delta i_{L}+\frac{\Delta i_{L}}{D T_{S}} t, & \text { if } 0 \leq t<D T_{S} \\
0, & \text { if } D T_{S} \leq t<T_{S}
\end{array}\right.
$$

where, $f_{s}=1 / T_{s}$ is the switching frequency, $\omega_{s}=2 \pi f_{s}$ is the angular switching frequency. $D$ is the duty cycle in steadystate. $I_{L}$ is the steady-state average value of the inductor current $i_{L} . \Delta i_{L}$ is the current ripple of $i_{L}$. The Fourier series of $i_{\text {in }}(t)$ contains harmonics at multiples of the switching frequency $f_{s}$, as follows:

$$
i_{\text {in }}(t)=\frac{a_{0}}{2}+\sum_{n=1}^{\infty} c_{n} \cos \left(\omega_{s} n t-\varphi_{n}\right)
$$

In our study, the harmonics multiple frequencies of $f_{s}$ are supposed to require a minor attenuation than the fundamental one at $f_{s}$. Then, the input filter is designed to smooth the busconnected current and reduce the first harmonic of the converter input current, to keep the bus-connected current ripple $\Delta i_{f}$ within the required constraints $\left(\Delta i_{f}<\Delta i_{f_{\text {lim }}}\right)$. The first harmonic coefficient $c_{1}$ of the Fourier series expansion can be expressed as:

$c_{1}=$
$\frac{\sqrt{\left(\left(\Delta i_{L}{ }^{2}+4 \pi^{2} D^{2} I_{L}{ }^{2}\right)\left(1-\cos ^{2}(\pi D)\right)+\pi^{2} D^{2} \Delta i_{L}{ }^{2} \cos ^{2}(\pi D)-\pi D \Delta i_{L}{ }^{2} \sin (2 \pi D)\right) / D^{2}}}{\pi^{2}}$

Then, the minimal cut-off angular frequency $\omega_{f_{\text {min }}}$ of the input filter has to respect the following condition:

$$
\operatorname{Att}_{D M}\left(f_{s}\right)=\left|\frac{\Delta i_{f_{l i m}}}{c_{1}}\right| \cong\left|\frac{\omega_{s}^{2}}{\omega_{f_{\text {min }}}^{2}}-1\right|^{-1}
$$

The input filter capacitance $C_{f}$, along with inductance $L_{f}$, define the value of the cut-off frequency $\omega_{f}$ and the attenuation of the filter:

$$
\omega_{f}=\left(L_{f} C_{f}\right)^{-\frac{1}{2}}
$$

Usually, to increase the attenuation of the filter, a smaller cutoff frequency is needed $\omega_{f} \leq \omega_{f_{\text {min }}}$, hence a bulkier filter (greater values of $L_{f}$ and $C_{f}$ ). Here, the objective is to smooth the bus-connected current and attenuate its ripple below than $0.1 \%$ of the maximum converter dc input current, i.e. $\Delta i_{f_{\text {lim }}}=$ $0.1 \%<i_{\text {in }}>_{T_{S}}$. Assuming the maximum load power is $3.5 \mathrm{~kW}$, the switching frequency is $20 \mathrm{kHz}$, the input voltage is $270 \mathrm{~V}$ and the output voltage is $200 \mathrm{~V}$. Therefore, $c_{1}=7.94 \mathrm{~A}$, $\omega_{f_{\text {min }}}=5107 \mathrm{rad} / \mathrm{s}$. To ensure that the input filter works effectively, the filter capacitance is chosen to $200 \mu \mathrm{F}$ first. Then, the filter inductance is selected to be $246 \mu \mathrm{H}\left(\omega_{f}=4508 \mathrm{rad} / \mathrm{s}\right)$.

\section{ACKNOWLEDGMENT}

The authors would like to thank the editors and reviewers for their useful comments and suggestions, which have helped the authors significantly improve the quality of this paper.

\section{REFERENCES}

[1] M. T. E. Heinrich, F. Kelch, P. Magne, and A. Emadi, "Regenerative Braking Capability Analysis of an Electric Taxiing System for a Single Aisle Midsize Aircraft," IEEE Trans. Transp. Electrific., vol. 1, no. 3, pp. 298-307, Oct. 2015

[2] J. Chen, C. Wang, and J. Chen, "Investigation on the Selection of Electric Power System Architecture for Future More Electric Aircraft," IEEE Trans. Transp. Electrific., vol. 4, no. 2, pp. 563-576, Jun. 2018.

[3] S. Pang, B. Nahid-Mobarakeh, S. Pierfederici, Y. Huangfu, G. Luo, and F. Gao, "Towards Stabilization of Constant Power Loads Using IDA-PBC for Cascaded LC filter DC/DC Converters," IEEE J. Emerg. Sel. Topics Power Electron., early access, Oct. 3, 2019, DOI: 0.1109/JESTPE.2019.2945331.

[4] Z. Ma, X. Zhang, J. Huang, and B. Zhao, "Stability-ConstrainingDichotomy-Solution-Based Model Predictive Control to Improve the Stability of Power Conversion System in the MEA," IEEE Trans. Ind. Electron., vol. 66, no. 7, pp. 5696-5706, Jul. 2019.

[5] H.-J. Kim, S.-W. Kang, G.-S. Seo, P. Jang, and B.-H. Cho, "Large-Signal Stability Analysis of DC Power System With Shunt Active Damper," IEEE Trans. Ind. Electron., vol. 63, no. 10, pp. 6270-6280, Oct. 2016.

[6] Y. Pan, L. Chen, X. Lu, J. Wang, F. Liu, and S. Mei, "Stability Region of Droop-Controlled Distributed Generation in Autonomous Microgrids," IEEE Trans. Smart Grid, vol. 10, no. 2, pp. 2288-2300, Mar. 2019.

[7] Y. Huangfu, et al. "Stability Analysis and Active Stabilization of Onboard DC Power Converter System with Input Filter." IEEE Trans. Ind. Electron., vol. 65, no. 1, pp. 790-799, Jan. 2018.

[8] L. Herrera, W. Zhang, and J. Wang, "Stability Analysis and Controller Design of DC Microgrids With Constant Power Loads," IEEE Trans. Smart Grid, vol. 8, no. 2, pp. 881-888, Mar. 2017.

[9] X. Zhang, B. Wang, U. Manandhar, H. Beng Gooi, and G. Foo, "A Model Predictive Current Controlled Bidirectional Three-Level DC/DC Converter for Hybrid Energy Storage System in DC Microgrids," IEEE Trans. Power Electron., vol. 34, no. 5, pp. 4025-4030, May 2019.

[10] M. A. Hassan, E. Li, X. Li, T. Li, C. Duan, and S. Chi, "Adaptive Passivity-Based Control of dc-dc Buck Power Converter With Constant Power Load in DC Microgrid Systems," IEEE J. Emerg. Sel. Topics Power Electron., vol. 7, no. 3, pp. 2029-2040, Sep. 2019.

[11] S. Pang et al., "Improving the Stability of Cascaded DC-DC Converter Systems via the Viewpoints of Passivity-Based Control and PortControlled Hamiltonian Framework," in 2019 IEEE Industry Applications Society Annual Meeting, Baltimore, MD, USA, 2019, pp. 1-6.

[12] M. K. Zadeh, R. Gavagsaz-Ghoachani, J.-P. Martin, S. Pierfederici, B. Nahid-Mobarakeh, and M. Molinas, "Discrete-Time Tool for Stability Analysis of DC Power Electronics-Based Cascaded Systems," IEEE Trans. Power Electron., vol. 32, no. 1, pp. 652-667, Jan. 2017.

[13] S. Rahmani, A. Rezaei-Zare, M. Rezaei-Zare, and A. Hooshyar, "Voltage and Frequency Recovery in an Islanded Inverter-Based Microgrid Considering Load Type and Power Factor," IEEE Trans. Smart Grid, vol. 10, no. 6, pp. 6237-6247, Nov. 2019.

[14] S. Pang, B. Nahid-Mobarakeh, S. Pierfederici, Y. Huangfu, G. Luo, and F. Gao, "Research on $L C$ Filter Cascaded with Buck Converter Supplying Constant Power Load Based on IDA-Passivity-Based Control," in IECON 2018 - 44th Annual Conference of the IEEE Industrial Electronics Society, Washington, DC, 2018, pp. 4992-4997.

[15] K.-W. Wang, X. Zhang, and H. S.-H. Chung, "Solid-State Single-Port Series Damping Device for Power Converters in DC Microgrid Systems," IEEE Trans. Power Electron., vol. 34, no. 1, pp. 192-203, Jan. 2019.

[16] R. W. Erickson and D. Maksimov, Fundamentals of Power Electronics. Norwell, MA, USA: Kluwer, 2001.

[17] M. Wu and D. D.-C. Lu, "A Novel Stabilization Method of $L C$ Input Filter With Constant Power Loads Without Load Performance Compromise in DC Microgrids," IEEE Trans. Ind. Electron., vol. 62, no. 7, pp. 4552-4562, Jul. 2015.

[18] L. Guo, S. Zhang, X. Li, Y. W. Li, C. Wang, and Y. Feng, "Stability Analysis and Damping Enhancement Based on Frequency-Dependent 
Virtual Impedance for DC Microgrids," IEEE J. Emerg. Sel. Topics Power Electron., vol. 5, no. 1, pp. 338-350, Mar. 2017.

[19] X. Zhang, Q.-C. Zhong, V. Kadirkamanathan, J. He, and J. Huang, "Source-Side Series-Virtual-Impedance Control to Improve the Cascaded System Stability and the Dynamic Performance of Its Source Converter," IEEE Trans. Power Electron., vol. 34, no. 6, pp. 5854-5866, Jun. 2019.

[20] X. Zhang, X. Ruan, and Q.-C. Zhong, "Improving the Stability of Cascaded DC/DC Converter Systems via Shaping the Input Impedance of the Load Converter With a Parallel or Series Virtual Impedance," IEEE Trans. Ind. Electron., vol. 62, no. 12, pp. 7499-7512, Dec. 2015.

[21] M. Karbalaye Zadeh, R. Gavagsaz-Ghoachani, S. Pierfederici, B. NahidMobarakeh, and M. Molinas, "Stability Analysis and Dynamic Performance Evaluation of a Power Electronics-Based DC Distribution System With Active Stabilizer," IEEE J. Emerg. Sel. Topics Power Electron., vol. 4, no. 1, pp. 93-102, Mar. 2016.

[22] E. Jamshidpour, B. Nahid-Mobarakeh, P. Poure, S. Pierfederici, F. Meibody-Tabar, and S. Saadate, "Distributed Active Resonance Suppression in Hybrid DC Power Systems Under Unbalanced Load Conditions," IEEE Trans. Power Electron., vol. 28, no. 4, pp. 1833-1842, Apr. 2013.

[23] R. Gavagsaz-Ghoachani, L.-M. Saublet, J.-P. Martin, B. NahidMobarakeh, and S. Pierfederici, "Stability Analysis and Active Stabilization of DC Power Systems for Electrified Transportation Systems, Taking into Account the Load Dynamics," IEEE Trans. Transp. Electrific., vol. 3, no. 1, pp. 3-12, Mar. 2017.

[24] X. Zhang, X. Ruan, H. Kim, and C. K. Tse, "Adaptive Active Capacitor Converter for Improving Stability of Cascaded DC Power Supply System," IEEE Trans. Power Electron., vol. 28, no. 4, pp. 1807-1816, Apr. 2013.

[25] S. Pang et al., "Interconnection and Damping Assignment Passivity-Based Control Applied to On-Board DC-DC Power Converter System Supplying Constant Power Load," IEEE Trans. Ind. Appl., vol. 55, no. 6, pp. 64766485, Nov. 2019.

[26] M. A. Hassan, E. Li, X. Li, T. Li, C. Duan, and S. Chi, "Adaptive Passivity-Based Control of DC-DC Buck Power Converter With Constant Power Load in DC Microgrid Systems," IEEE J. Emerg. Sel. Topics Power Electron., vol. 7, no. 3, pp. 2029-2040, Sep. 2019.

[27] S. Pang, B. Nahid-Mobarakeh, S. Pierfederici, Y. Huangfu, G. Luo, and F. Gao, "Large-Signal Stable Nonlinear Control of DC/DC Power Converter with Online Estimation of Uncertainties," IEEE J. Emerg. Sel. Topics Power Electron., early access, July. 21, 2020, DOI: 10.1109/JESTPE.2020.3010895.

[28] J. Zeng, Z. Zhang, and W. Qiao, "An interconnection and damping assignment passivity-based controller for a DC-DC boost converter with a constant power load," IEEE Trans. Ind. Appl., vol. 50, no. 4, pp. 2314 2322, Jul./Aug. 2014.

[29] Y. Meng, S. Shang, H. Zhang, Y. Cui and X. Wang. "IDA-PB Control with Integral Action of Y-connected Modular Multilevel Converter for Fractional Frequency Transmission Application," IET Gener. Transm. Distrib., vol. 12, no. 14, pp. 3385-3397, Aug. 2017.

[30] Y. Gui, B. Wei, M. Li, J. M. Guerrero, and J. C. Vasquez, "Passivity-based Coordinated Control for Islanded AC Microgrid," Applied Energy, vol. 229, pp. 551-561, Nov. 2018.

[31] R. V. Meshram, "Port-Controlled Phasor Hamiltonian Modeling and IDAPBC Control of Solid-State Transformer," IEEE Trans. Control Syst. Technol., vol. 27, no. 1, pp.161-174, Jan. 2019.

[32] R. Ortega, A. J. van der Schaft, B. Maschke, and G. Escobar, "Interconnection and Damping Assignment Passivity-Based Control of Port-controlled Hamiltonian Systems," Automatica, vol. 38, no. 4, pp. 585-596, Apr. 2002. 